\title{
Wheat Genetic Loci Conferring Resistance to Yellow Rust in the Face of Recent Epidemics of Genetically Diverse Races of the Fungus Puccinia Striiformis F. Sp. Tritici
}

\section{Laura Bouvet}

NIAB: National Institute of Agricultural Botany

\section{Lawrence Percival-Alwyn}

NIAB: National Institute of Agricultural Botany

\section{Simon Berry}

Limagrain UK Ltd.

Paul Fenwick

Limagrain UK Ltd.

Camila Campos Mantello

NIAB: National Institute of Agricultural Botany

\section{Sarah Holdgate}

NIAB: National Institute of Agricultural Botany

lan Mackay

NIAB: National Institute of Agricultural Botany

James Cockram ( $\square$ James.Cockram@niab.com )

NIAB https://orcid.org/0000-0002-1014-6463

\section{Research Article}

Keywords: Bread wheat (Triticum aestivum L.), yellow rust, stripe rust, quantitative trait loci (QTL), multiparent advanced generation inter-cross (MAGIC), marker-assisted selection (MAS)

Posted Date: April 27th, 2021

DOI: https://doi.org/10.21203/rs.3.rs-459064/v1

License: (c) (1) This work is licensed under a Creative Commons Attribution 4.0 International License. Read Full License 
1 Wheat genetic loci conferring resistance to yellow rust in the face of recent epidemics of

2

3

4

5

6

7

8

9

10

11

\section{genetically diverse races of the fungus Puccinia striiformis f. sp. tritici}

Laura Bouvet $^{1,2}$, Lawrence Percival-Alwyn ${ }^{1}$, Simon Berry ${ }^{3}$, Paul Fenwick ${ }^{3}$, Camila Campos Mantello $^{1}$, Sarah Holdgate ${ }^{1}$, Ian J. Mackay ${ }^{1 \dagger}$, James Cockram ${ }^{1 *}$

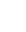

${ }^{1}$ NIAB, 93 Lawrence Weaver Road, Cambridge, CB3 OLE, United Kingdom

${ }^{2}$ Department of Plant Sciences, University of Cambridge, Downing Street, Cambridge, CB2 3EA, United Kingdom

${ }^{3}$ Limagrain UK Ltd, Market Rasen, LN7 6DT, United Kingdom

${ }^{\dagger}$ Current address: SRUC, The King's Buildings, West Mains Road, Edinburgh, EH9 3JG, United Kingdom

*Corresponding author: james.cockram@niab.com. ORCID: http://orcid.org/0000-0002$1014-6463$ 


\section{Abstract}

Yellow rust (YR), or stripe rust, is a major fungal disease of wheat (Triticum aestivum) caused by Puccinia striiformis f. sp. tritici (Pst). Since 2011, the historically clonal European Pst races have been superseded by the rapid incursion of genetically diverse lineages, reducing the resistance of varieties that previously showed durable resistance. Identification of sources of genetic resistance to such races is a high priority for wheat breeding. Here we use a wheat eight-founder multi-parent population genotyped with a 90,000 feature single nucleotide polymorphism array to genetically map adult plant YR resistance to such new Pst races. Analysis of five trials, at three sites in the UK, consistently identified four highly significant quantitative trait loci (QTL) across all test environments, located on chromosomes 1A (QYr.niab-1A.1), 2A (QYr.niab-2A.1), 2B (QYr.niab-2B.1) and 2D (QYr.niab-2D.1). Together these explained $\sim 50 \%$ of the phenotypic variation, and genetic markers were developed that distinguished resistant and susceptible alleles. Analysis of these QTL in two-way and threeway combinations showed combinations conferred greater resistance than single QTL. Four additional major-effect QTL were detected in two or more trials, together explaining 15-20\% of the phenotypic variation, as well as six minor QTL. Genomic analysis found the median physical interval size of these eight QTL to be $19.8 \mathrm{Mbp}$, and QYr.niab-2A.1 and QYr.niab$2 D .1$ to be at homoeologous locations on the group-2 chromosomes. Notably, the QYr.niab2B.1 physical interval contained five nucleotide-binding leucine-rich repeat (NLR) candidate genes with integrated BED domains, of which two corresponded to the cloned resistance genes $Y r 7$ and $Y r 5 / Y r S p$.

\section{Key message}

Genetic analysis of a wheat multi-founder population identified 14 yellow rust resistance QTL. For the four most significant QTL, additive effects were demonstrated, and genetic markers validated for marker-assisted selection. 


\section{Introduction}

Yellow rust (YR), caused by the biotrophic fungus Puccinia striiformis f. sp. tritici (Pst), is a widespread pathogen of wheat (Triticum aestivum L.), and a substantial threat to global wheat production. Since the 2000 s, a subset of genetically diverse and divergent $P$ st lineages have been responsible for recurrent YR epidemics in numerous wheat-producing regions (Ali et al. 2014; 2017). The rapid adaptation and subsequent spread of these lineages into previously hostile environments has given rise to more aggressive pathotypes that are generally better adapted to higher temperatures (Milus et al. 2009). In the United Kingdom (UK) and NorthWestern Europe, the historically clonal Pst population has been largely displaced by a genetically diverse group of lineages (Hubbard et al. 2015; Hovmoller et al. 2016). First detected simultaneously in 2011 across several European countries, the 'Warrior' and 'Kranich' Pst races likely originated in the near Himalayan region and rapidly spread throughout the continent as a group of genetically distinct lineages. The ability of Pst to migrate over long distances, to locally adapt to new environments, and for new lineages to rapidly displace established populations, has had a notable impact on the resistant levels of wheat varieties in the UK (Hubbard et al. 2015; Bueno-Sancho et al. 2017) and beyond (Wellings, 2011; Hovmøller et al. 2016), prompting a review of YR resistance breeding and deployment strategies.

The most efficient way to control the effects of wheat fungal disease is via approaches that combine agricultural and agronomic practices, disease monitoring and genetic improvement of the wheat varieties grown (Downie et al. 2020). Resistance breeding focuses on two classes of rust resistance $(R)$ genes. The first, termed 'seedling resistance' or 'all stage resistance', confers qualitative resistance, typically to one or a few Pst isolates. The second, termed 'adult plant resistance', typically provides quantitative resistance against multiple pathogen races. Over 300 genomic regions conferring YR resistance in wheat have been reported (Rosewarne et al. 2013; Wang \& Chen, 2017). Of these, approximately 80 are permanently named yellow rust resistance $(Y r)$ genes, recently summarised by Jamil et al. (2020). To date, $16 R$ genes controlling seedling resistance to different wheat rust pathogens have been cloned, and all encode nucleotide-binding leucine-rich repeat (NLR) proteins: Lrl (Cloutier et al. 2007), Lr10 (Feuillet el al. 2003), Lr21 (Huang et al. 2003), Lr22a (Thind et al. 2017), Rga2 (Loutre et al. 2009), Sr13 (Zhang et al. 2017), Sr22 (Steuernagel et al., 2016), Sr33 (Periyannan et al. 2013), Sr35 (Saintenac et al. 2013), Sr45 (Steuernagel et al. 2016), Sr46 and SrTA1662 (Arora et al. 
2019), Sr50 (Mago et al. 2015), $Y r 7$ and $Y r 5 / Y r S P$ (Marchal et al. 2018). Similarly, wheat $R$ genes to other fungal pathogens are also encoded by $N L R$ genes, such as the powdery mildew resistance genes Pm2 (Sánchez-Martin et al. 2016), Pm3b (Yahiaoui et al. 2004), Pm8 (Hurni et al. 2013), Pm21 (He et al. 2018) and Pm41 (Li et al. 2020). Host NLR proteins predominantly act by recognising the effector molecules that pathogens produce to inhibit host defence responses (Jones et al. 2016). To detect the large range of potential infecting pathogens, plant NLR gene families have radiated and diversified, commonly by localised gene duplication, unequal crossing-over, and variation within the leucine-rich repeat (LRR) domains which bind pathogen effectors (Sarris et al. 2016). Some NLRs have evolved to include a variety of additional 'integrated' domains that may be involved in receptor activation or downstream signalling (Sarris et al. 2016), most commonly kinase and DNA-binding domains (Anderson et al. 2020; Steuernagel et al. 2020). Examples include the BED zinc finger domain in $\mathrm{Yr} 7$ and two alleles at a closely located paralogous gene termed $\operatorname{Yr} 5$ and $\operatorname{YrSp}$ (Marchal et al. 2018), as well as the serine/threonine protein kinase (S/TPK) domain encoded by the wheat gene Tsn1 conferring sensitivity to the necrotrophic fungal pathogens that cause tan spot and Septoria nodorum blotch (Faris et al. 2010). NLRs can also act by monitoring the status of host receptor proteins, termed 'indirect recognition'. For example, the Arabidopsis NLRs RPM1 and RPS2 monitor RPM1-interacting protein 4 (RIN4) for cleavage or phosphorylation by several bacterial effectors (Mackey et al. 2002; Axtell et al. 2003; Andersson et al. 2006). RIN4 acts as a susceptibility factor, but is also essential for normal plant growth (Mackey et al. 2002). Recently a wheat YR susceptibility gene encoding a branched-chain amino acid aminotransferase, TaBCAT1, has been identified via analysis of genes upregulated after $P s t$ infection (Corredor-Moreno et al. 2021), although its mutation as single and double mutants in tetraploid wheat was not reported as affecting non-disease phenotypes. In contrast to seedling resistance genes, each of the three map-based cloned YR adult plant resistance genes encode a different class of protein: Yr18/Lr34 an ABC transporter (Krattinger et al. 2009), Yr36 a kinaseSTART domain protein (Fu et al. 2009), Yr46/Lr67 a hexose transporter (Moore et al. 2015) and $\mathrm{Yr15/YrG303/YrH52}$ a kinase-pseudokinase protein (Klymiuk et al. 2018; 2020). Wheat breeding strategies historically focused on the use of qualitative yellow rust resistance genes, sometimes in isolation. $\operatorname{Yr} 17$ for example was a popular source of such resistance in NorthWestern Europe. The effectiveness of the genetic resistance conferred by $\operatorname{Yr} 17$ stopped soon after its deployment as a single resistance gene over a large wheat acreage (Bayles et al. 2000), although more recently this locus, or one genetically linked to it, provides resistance against Warrior Pst isolates. Where possible, resistance breeding strategies now favour the more 
durable approach of combining race specific and non-race specific resistance genes to provide broad-spectrum resistance (Singh et al. 2005; Chen et al. 2013). Such use of genetic resistance will continue to be aided by the genetic and molecular characterisation of $Y r$ genes and quantitative trait loci (QTL), understanding of how these genetic loci interact with each other, and molecular markers to track favourable alleles and allelic combinations in breeding programmes.

YR resistance QTL and $Y r$ genes have predominantly been identified in biparental (e.g. Rosewarne et al. 2013) and association mapping panels (e.g. Maccaferri et al. 2015; Zegeye et al. 2014; Kertho et al. 2015). The crossing of just two parents in a biparental population inevitably limits the number of resistance genes and alleles that can be investigated (Mackay, 2001). While association mapping panels overcome this limitation by exploiting historical recombination events in the germplasm collection used, population structure in such collections can lead to false marker-trait associations (Cockram \& Mackay 2018). Multi-founder populations (reviewed by Cockram \& Mackay, 2018; Scott et al. 2020) now provide complementary resources for the genetic investigation of wheat disease resistance. Such designs include multiparent advanced generation inter cross (MAGIC) populations, derived by inter-crossing all founders over multiple generations before the generation of inbred lines. As each MAGIC progeny line likely contains alleles from all founders dispersed throughout its genetic background, MAGIC populations allow the effects of multiple alleles to be assessed within a single unified population. We previously developed the eight-founder 'NIAB Elite MAGIC' population, estimated to capture $>80 \%$ of the genetic variation observed in UK wheat based on single nucleotide polymorphism (SNP) analysis (Mackay et al. 2014). Here we analyse this MAGIC population for resistance to YR at five trials conducted over two seasons and three sites in the United Kingdom (UK). Eight major adult plant resistance QTL were resolved, identified across growth seasons in which the new genetically diverse 'Warrior' group of Pst races were endemic in the UK. The gene space of these genetic loci was explored, candidate genes identified, and genetic markers tagging resistant haplotypes validated for the four most significant QTL. Additionally, six minor effect QTL were identified. Collectively, this work highlights the role of combinations of adult plant YR resistance genes in the genetic control of the aggressive Pst pathotypes that have replaced the previous asexual populations of the pathogen. 


\section{Methods}

\section{Germplasm, trial design, and field trials}

The 'NIAB Elite MAGIC' wheat population (Mackay et al. 2014) consists of eight founders (the winter varieties Alchemy, Brompton, Claire, Hereward, Rialto, Robigus, Soissons, and the facultative variety Xi19) inter-crossed over three generations, and the outputs of the crossing then selfed over multiple generations to produce $>1,000$ recombinant inbred lines (RILs). The population was grown in five field trials over two seasons (YR assessment seasons 2015 and 2016) at three sites in the UK: (1) NIAB-Cambridge trial ground (latitude 52.235010, longitude 0.097871), Osgodby (latitude 53.410161, longitude -0.386770) and Rothwell (latitude 53.482597, longitude -0.259779). All trials followed an incomplete randomised block design generated with the DEW experimental design software, formerly www.expdesigns.co.uk but superseded by the R package 'blocksdesign' (Edmundson, 2020). The numbers of MAGIC RILs and control lines assessed at each site and year combination is listed in Table 1 and Supplementary Table 1. Seed for each season's trials were sourced from nursery plots grown the preceding season. Accordingly, RIL 9 and RIL 10 seed were used for the 2015 and 2016 season trials, respectively. Standard agronomy practices were used for commercial wheat production at each location, but lacking application of chemical protection against YR. All trials were Autumn sown in the calendar year preceding YR assessment. Each line was sown in two 1-m rows, with six rows per plot. At the NIAB trial ground in Cambridgeshire, the central two rows were sown with the spreader wheat variety Vuka, known to be highly susceptible to all known UK races of YR, with RILs on either side. At the Osgodby and Rothwell sites in Lincolnshire, the central two rows were left empty. Instead, Vuka was present as a whole plot every three traverses. All trials apart from OSG16 were inoculated with a mixture of $P$ st races ('Solstice race' isolate 08/21 virulent on $\operatorname{Yr} 1,2,3,4,6,9,17,25,32$ and 'Warrior race' isolate 11/08 virulent on $\operatorname{Yr} 1,2,3,4,6,79,17,25,32, S p)$. Trial OSG16 was uninoculated, and therefore exposed only to natural YR infection.

\section{Phenotypic data}

YR infection severity of the leaves was assessed as the percentage of total leaf tissue with sporulating uredinia, estimated using the modified Cobb's scale (Peterson et al. 1948) ranging from 0 to $100 \%$ infection (\%inf). Leaf infection severity was assessed on three to four occasions at 12-18 day intervals at each trial, from the end of booting (Zadocks growth stage 45-49) until the mid-to-hard-dough stage (growth stage 85-87). 
Trials analysis

A stepwise model selection approach was used to estimate the Best Linear Unbiased Estimators (BLUEs) for MAGIC RILs, integrating spatial and non-spatial mixed methods based on

199 Restricted Maximum Likelihood (REML) implemented in Genstat, $18^{\text {th }}$ edition (VSN International, 2015). Three models were considered: Model 1 - Blocking (genetic effects are estimated based only on the inter- and intra-block variation recovered from the model); Model 2 - Spatial (only considers global and/or local field trends); Model 3 - Spatial + blocking (combination of the above models). Initially, each model was optimised by including field trends running in either row or column direction or scoring order (the route used to score the trial), followed by between-model comparison to select the one that best fits the data. Further details of the three models used is given in Supplementary Text 1. The Akaike Information Criterion (AIC) was used as a measure for model selection (Akaike, 1974). Each model was optimised using AIC as a measure of model fit improvement, and the model with the lowest AIC value selected. Natural log transformation of the disease severity data was performed in cases where residuals were observed not to be normally distributed (trials NIAB16 and OSG16), to improve the normality of the residuals. These were assessed visually with histograms illustrating the distribution of the residuals and Q-Q normality plots. The ShapiroWilk normality test was used to further support these observations. Broad sense heritability $\left(h^{2}\right)$ was used as a measure of total phenotypic variation attributable to the genotypic effect. The VHERITABILITY function in Genstat $\left(18^{\text {th }}\right.$ edition, VSN International 2015) was used to calculate $h^{2}$ for each trial, and is based on the definition of heritability given by Cullis et al. (2006) and Piepho et al. (2007).

\section{Genetic analysis}

Genetic analysis was carried out using the 7,369 SNPs mapped to unique positions on the MAGIC genetic map (Gardner et al. 2016). For all trials, two broad methods were used for genetic analysis. (1) Single marker analysis (SMA): regression against allelic state at single markers using R/lme4 (Bates et al. 2015) in R (R Core Team, 2017) using the following mixed model:

$Y=\mu_{x}+G_{m}+\beta+e$ where $Y$ is the YR resistance value, $\mu$ is the adjusted YR score for MAGIC RIL $x, G_{m}$ is the fixed SNP marker effect, $\beta$ is the population structure consisting of 'funnels' and 'plants within 
funnels' effects (from Mackay et al. 2014), and $e$ as the residual error term. The model has one degree of freedom, since regression is carried out on binary allelic state. Multiple-test correction was carried out using R/qualue (Storey 2015), with a threshold of $q<0.05$. (2) Haplotype-based analysis, for which founder haplotype probabilities were calculated with the mpprob function in R/mpMap (Huang et al. 2011) with a threshold of 0.5. Three types of haplotype-based analyses were conducted. Identity by descent (IBD): regression against haplotype probability estimates using R/qtl and the following mixed model:

$Y=\mu+G_{p}+\beta+e$

where $Y, \mu, \beta$ and $e$ are as for SMA above, and $G_{p}$ is the fixed term for founder probabilities. Here, the statistical model has up to seven degrees of freedom. A QTL significance threshold $q<0.05$ was used. Interval mapping (IM): conducted in $\mathrm{R} / \mathrm{mpMap}$ using the haplotype probability estimates. Composite interval mapping (CIM): conducted in R/mpMap with 10 covariates using the haplotype probability estimates. Within mpMap, an automated forward selection process based on AIC values was used to select the best ten marker covariates for each MAGIC line. Significant QTLs were then selected in two stages. First, mpMap scans the 100 markers surrounding a particular marker location and selects QTLs based on a threshold of $-\log 10(p)>3$. The number of significant QTL were then reduced by fitting a model with $p<0.05$ and with phenotypic variance explained (PVE) $>0.5 \%$. Significance threshold was estimated based on the simulation of the null distribution. Additionally, R/mpMap outputs founder contributions for each significant QTL, computed using a regression approach at each marker location. To summarise the results of the four analyses, the $p$ or $q$ values from each mapping method for all adjusted and log transformed YR scores, and for all environments, were compiled into a single table and a 'consensus' peak marker for each QTL identified following the methods described in Supplementary Text 2. 'Major' and 'minor' QTL were defined as those explaining either $>5 \%$ or $<5 \%$ of the phenotypic variance, respectively.

\section{Bioinformatic analysis}

The locations of the genetically mapped MAGIC SNPs on the physical map were determined using the SNP flanking DNA sequences (Wang et al. 2014) as queries for BLASTn (Altschul et al. 1990) interrogation of the wheat reference genome assembly (IWGSC, 2018). Where BLASTn hits of equal match were identified on more than one chromosome, genetic map position (Gardner et al. 2016) was used to assign BLASTn hits to chromosomes. For analysis of the YR QTL physical intervals in the context of disease resistance gene density, likely 
resistant genes were first identified from the IWSGC RefSeq v1.0 assembly (IWSGC, 2018) high- and low-confidence gene functional annotations from v1.0 mapped on to gene model annotation RefSeq v1.1 (Alaux et al. 2018) where transcript.1 contained any of the following search terms: 'NB-LRR'; 'NBS-LRR'; 'NB-ARC'; 'TIR-NBS'; 'LRR family protein'; 'Leucine-rich repeat domain'; 'Plant disease resistance response'; 'Nucleotide binding site leucine-rich repeat'; 'disease resistance protein (TIR class)'. The percentile ranks of all gene counts and resistant gene counts for each QTL were calculated using windows of the same lengths $(9.85$ - $133.91 \mathrm{Mbp})$ sampling the genome (all 21 chromosomes plus the unassigned chromosome) every $100 \mathrm{bp}$. Plots were generated using the Circos visualization tool (Krzywinski et al. 2009). QTL resistant gene enrichment $p$-values were calculated using the binomial cumulative probability function where the chance of success is 0.365 (genome wide, resistant genes / total genes), the number of tests is the number of genes within the QTL and the number of successes is the number of resistant genes within the QTL.

\section{Development of KASP markers}

Selected SNPs from the 90k array were converted to the Kompetitive Allele-Specific PCR (KASP) genotyping system. SNP genomic flanking sequences were obtained from the T3 Wheat website (https://triticeaetoolbox.org/wheat/) and used as queries for KASP primer design using Polymarker (Ramirez-Gonzalez et al. 2015) with additional manual curation. The resulting primers (Supplementary Table 2) were used for KASP genotyping as described by Downie et al. (2018), with genomic DNA from the eight MAGIC founders extracted using a DNeasy Plant Mini Kit (Qiagen). Haplotypes were constructed using five SNPs either side of the peak marker for each QTL and markers best able to discriminate between these haplotypes selected. For QTL QYr.niab-2D.1, difficulties in successfully converting SNPs to KASP meant that a wider window of SNPs was considered. 
YR infection was assessed in the MAGIC population (Figure 1) grown across five trials in the UK, with phenotyping conducted at two timepoints per site. Three of the trials were conducted in 2015 (sites NIAB15, OSG15 and ROTH15) and two in 2016 (NIAB16 and OSG16). In 2016, YR scores were skewed towards the resistant end of the scoring scale, and a normal distribution was not observed. Normality for the 2016 season trials was improved using the natural log transformation, and so was used in all subsequent analyses that year. Normality tests on the 2015 season trials found the residuals to all be normally distributed, and so no further transformation was required. To account for variation within the field, three linear model approaches were applied to the 2015 raw data and the $2016 \log$ transformed data, and the best models selected via Akaike Information Coefficient (AIC) (Supplementary Table 3). YR phenotypic data is listed in Supplementary Table 4.

Broad sense heritability was high across all test environments $\left(h^{2}=0.93-0.95\right)$ (Supplementary Table 3). YR infection scores in the MAGIC founders spanned from 0 to $100 \%$, with similar trends in overall founder ranking observed between sites and years (Figure 2A). Soissons (0-3 $\%$ infection) and Robigus (70-100 \% infection) were the most resistant and susceptible parents respectively, regardless of year, site and scoring timepoint. While the remaining parents varied somewhat in ranking dependent on year and location, two main founder groups were observed: relatively resistant (percentage infection range: 3-33; mean $=10.4$ : Alchemy, Hereward and Xi19) or relatively susceptible (percentage infection range: $17-76$; mean $=36.8$ : Brompton, Claire and Rialto). The ranking of varieties within a trial remained the same across the two scoring timepoints. For the MAGIC RILs, YR scores were similarly distributed at the different sites within each year, but differed considerably between years (Figure 2B). In the RILs, transgressive segregation was observed in 2015 and 2016, both above and below that observed for the most susceptible and resistant founders, respectively (Figure 2B). In 2015, as YR progressed through the season and susceptibility increased among the MAGIC population, a subset of 31 RILs remained highly resistant (\%inf < 1$)$ at all three sites. Intermediate YR scores (10-80 \% inf) were normally distributed, with this trend more evident at OSG15 and ROTH15, where $P s t$ developed more gradually compared to at NIAB15. In 2016, percent YR infection was more skewed towards resistance, compared to the previous year. High levels of resistance (\%inf < 1) were maintained by 32 RILs, of which eight were the same as in 2015. Overall, 121 
and 171 MAGIC lines exhibited a resistant response (\%inf <10) throughout the 2015 and 2016 scoring seasons respectively, at all three locations.

\section{Genetic mapping of yellow rust resistance in MAGIC}

Overall, 14 YR adult plant resistance QTL we identified (Table 2). These could be broadly divided into two groups: eight 'major' QTL that explained $>5 \%$ of the phenotypic variance, were highly significant ( $p>1.0 \mathrm{E}-05)$ and identified in all trial/score combinations, and six 'minor' QTL that explained less than 5\% of the variance and had lower significance $(p<1.0 \mathrm{E}-$ 05) (Tables 3-4). The eight 'major' QTL were robustly replicated, identified in all five test environments. While six of these 'major' QTL were identified via three or more genetic analysis methods, QYr.niab-2A.2 and QYr.niab-6A.2 were identified using IBS and IBD alone. By far the most significant QTL were located on the long arms of chromosomes 2B (QYr.niab2B.1) and 2D (QYr.niab-2D.1) (Figure 3; Table 3), each of which had minimum $p$-values $<2.22 \mathrm{e}^{-16}$ and explained up to $\sim 18 \%$ of the phenotypic variance. Predicted allelic effects at QYr.niab-2B.1 showed Soissons to carry the most resistant allele, while for QYr.niab-2D.1, Alchemy and Claire alleles conferred the highest resistance (Table 4). The four 'major' QTL on chromosomes 1A, 2A and 3A, were found to be the next most significant YR resistance loci, with $p$-values $\leq 1.41 \mathrm{e}^{-07}$. Of these, the founders Claire and Hereward conferred the strongest resistance at QYr.niab-1A.1, Rialto and Xi19 at QYr.niab-2A.1, and Hereward and Rialto at QYr.niab-3A.1 (Table 4). As QYr.niab-2A.2 and QYr.niab-6A.2 were detected using single marker analysis only, no founder effects were calculated. The two remaining 'major' QTL were located on chromosome $6 \mathrm{~A}$, had $p$-values $\leq 1.22 \mathrm{e}^{-05}$, and explained lower amounts $(\sim 5-6 \%)$ of the phenotypic variance. At QYr.niab-6A.1, Xi19 conferred the most resistant allele, while for QYr.niab-6B.1 alleles from Brompton, Rialto and Xi19 conferred resistance.

Additionally, six 'minor' QTL were identified, located on chromosomes 3D (QYr.niab-3D.1), 4B (QYr.niab-4B.1), 4D (QYr.niab-4D.1), 5A (QYr.niab-5A.1), 6A (QYr.niab-6A.3) and 6B (QYr.niab-6B.1) (Table 3). These each explained a relatively low percentage of the phenotypic variance, ranging from 2.49 to $4.08 \%$. The majority of these 'minor' QTL were detected in lower numbers of test environments, although QYr.niab-6A.3 was detected in all but one site/score combinations analysed. QYr.niab-4B.1 was only detected in the 2016 season trials and was the only one of the 14 YR QTL identified not to have been replicated between the two seasons. 


\section{Pairwise QTL interactions}

MAGIC RILs were divided into 16 different groups, based on the presence or absence of resistance alleles at the four most significant YR QTLs ( $p<0.0001$, PVE $>8 \%$ : QYr.niab-1A.1, QYr.niab-2A.1, QYr.niab-2B.1 and QYr.niab-2D.1) (Figure 4). For each QTL, calling of the resistant allele was based on the allelic state of the consensus peak markers for each MAGIC founder. A one-way ANOVA found significant differences in the YR disease severity between all the resulting QTL combinations $\left(p<2.2 \mathrm{E}^{-16}\right.$, Table 5). This analysis was followed by a pairwise $t$-test for the comparison of $\%$ disease severity means (Supplementary Table 5). Stacking resistance alleles at all three-way QTL combinations were among the most effective in significantly reducing MAGIC RIL YR infection and displayed high resistance responses to YR infection ( $t$-test values $<2.0 \mathrm{E}^{-16}$ ). Notably, combining resistance alleles at QYr.niab-1A.1, QYr.niab-2B.1 and QYr.niab-2D.1 conferred near complete resistance across all trial sites (Figure 4). Where resistance at QYr.niab-2D.1 was combined with resistance from QYr.niab$1 A .1$ or $Q Y$ r.niab-2B.1, the resulting two-way combinations showed significant reductions in YR susceptibility, comparable to some of the three-way combinations ( $t$-test values $\left.<2.0 \mathrm{E}^{-16}\right)$. All other combinations were also significant in reducing disease severity $\left(4.8 \mathrm{E}^{-16}<t\right.$-test values $>2.4 \mathrm{E}^{-15}$ ). Single QTL still provided significant levels of resistance against YR but were less effective compared to combinations (QYr.niab-2D.1: $t$-test value $=1.3 \mathrm{E}^{-13}, Q Y$ r.niab-1A.1: $t$ test value $=4.3 \mathrm{E}^{-10}$ and $Q Y r . n i a b-2 A .1: t$-test value $\left.=1.8 \mathrm{E}^{-05}\right)$. The combination of $Q Y$ Yr.niab-1A.1 and QYr.niab-2B.1 was the only one found to not significantly reduce disease severity ( $t$-test value=0.04). Year and site effects were observed for the QTL combinations QYr.niab1A.1/QYr.niab-2A.1 and QYr.niab-1A.1/QYr.niab-2B.1. In both cases, the 2016 trial sites exhibited a narrower range of disease severity response compared to the 2015 sites. Similarly, the QYr.niab-2A.1/QYr.niab-2B.1 combination was less effective in conferring YR resistance at OSG15 compared to the other trial sites. Notably, the 82 highly YR resistant MAGIC RILs (disease severity <10\%) resistant across all trial sites exhibited all of the 16 QTL combinations investigated, apart from the four-way combination (1A, 2A, 2B, 2D) and QYr.niab-1A.1 present alone.

\section{Analysis of physical map locations of yellow rust resistance $Q T L$}

Anchoring QTL flanking markers to the wheat reference genome found the physical intervals of the eight major and minor YR QTL identified to range from 9.852 Mbp for QYr.niab-3A.1 to $133.909 \mathrm{Mbp}$ for QYr.niab-2A.1 (median = $19.839 \mathrm{Mbp}$ ) (Supplemental Table 6). To investigate the genomic locations these QTL further, scatterplots of genetic (Gardner et al. 
2016) versus physical (IWGSC, 2018) map locations were created for the six relevant chromosomes (1A, 2A, 2B, 2D, 3A, 6A), and the locations of the peak SNPs for each QTL highlighted (Figure 5). While at least half of each of these six chromosomes were located in regions of very low genetic recombination spanning the centromere, all eight major YR resistance QTL were located outside of these regions. Indeed, three of the eight major QTL were located $<18 \mathrm{Mbp}$ from the chromosome telomeres in regions with high genetic recombination (QYr.niab-2A.2, QYr.niab-2D.1 and QYr.niab-6A.1), two were located on the shoulders of the non-recombining regions (QYr.niab-2A.1, QYr.niab-2B.1), with the remainder located in the intervening regions within which medium levels of genetic recombination typically occur. Of note, four QTL were located in broadly homoeologous (i.e. colinear) genomic locations: the 'major' QTL QYr.niab-2A.2 and QYr.niab-2D.1 on the long arm of the homoeologous chromosomes 2A and 2D, respectively, and the 'major' QTL QYr.niab-6A.2 was colinear with 'minor' QTL QYr.niab-6B.1 (Supplementary Table 6).

Next, using the wheat reference genome assembly and gene model annotation, we investigated the genomic locations of the 14 YR QTL in respect to overall gene density, and to the density of genes belonging to the NBS-LRR gene family (Figure 6; Supplementary Table 6). Seven of the 14 QTL were located in genomic regions that contain very high numbers of genes $\left(>95^{\text {th }}\right.$ percentile, genome wide). Additionally, four QTL were located in regions with significantly higher number of 'resistance genes', based on simple NBS-LRR gene count ( $>95^{\text {th }}$ percentile, genome wide). Furthermore, six QTL had a significantly higher number of 'resistance genes' than expected from the QTL gene count, compared to as expected genome-wide, calculated using the binomial cumulative probability function $(P>0.05)$. Details of potential candidate genes within the QTL physical intervals, based on gene model functional annotations and predicted protein domains, as well as sequence searches using the 15 wheat rust $R$ genes cloned to date (Supplemental Table 7), are detailed in Supplementary Table 8. In addition to NLR genes characteristic of seedling resistance genes, the colinear QYr.niab-2A.2 and QYr.niab$2 D .1$ were notable for the prominence of receptor-like kinase candidate genes, which encode proteins with protein-kinase and LRR domains. Similarly, the broadly colinear QYr.niab-6A.2 and QYr.niab-6B.1 QTL both had high numbers of F-box/LRR domain encoding genes in additional to multiple copies of canonical NLRs, with QYr.niab-6B.1 also containing a PKNLR (TraesCS6B02G099900; although its 6A homoeologue TraesCS6A02G093400LC is a pseudogene and so not annotated as a PK-NLR). QYr.niab-2B.1 was found to span the wheat reference genome gene models reported to be homologous to the cloned wheat YR resistance 
genes $Y r 7$ and $Y r 5 / Y r S P$, represented in the reference genome assembly of cv. Chinese Spring by gene models TraesCS2B02G488000 and TraesCS2B02G488600/TraesCS2B02G488700, respectively. Both $Y r 7$ and $Y r 5 / S P$ encode NLRs with an integrated BED domain, with three additional BED-NLR candidate genes located very close-by (TraesCS2B02G488400, TraesCS2B02G734100LC and TraesCS2B02G48900). With the exception of $Y r 7$ and $\mathrm{Yr} 5 / \mathrm{YrSP}$, none of the remaining 13 cloned wheat $R$ genes, or genes with high sequence similarity to these $R$ genes, were located in the QTL intervals identified here.

\section{Development of KASP markers tagging resistant haplotypes}

For the four most robust YR QTL investigated above, we used SNPs from the 90k SNP array able to discriminate resistant and susceptible haplotypes or SNPs (Supplementary Table 2) and converted these to the KASP genotyping platform (Figure 7). For QYr.niab-1A.1, two SNPs (tplb0021i12_383 and BobWhite_c44164_402) defined three haplotypes and were successfully converted to co-dominant KASP markers. Resistant haplotypes 1A-H1_0.0 and 1A-H2_2.0 uniquely identified the resistant alleles present in Hereward and Soissons, respectively. However, as the remaining haplotype 1A-H3_2.0 was not able to discriminate between the predicted resistant allele(s) from Claire and Xi19 from the susceptible alleles conferred by the remaining four founders, we were not able to discriminate between all of the predicted alleles at the locus. For QYr.niab-2A.1, two SNPs were converted to co-dominant KASP markers: BS00062679_51 and BS00022641_51. These defined three haplotypes at the locus, with haplotype 2A-H1_2.0 unique to the resistant alleles present in Rialto and Xi19. The remaining two haplotypes tagged susceptible founder alleles: 2A-H2_2.2 (Alchemy, Brompton, Claire, Hereward, Robigus) and 2A-H30.2 (Soissons). For QYr.niab-2B.1, SNP BS00016650_51 was converted to a co-dominant KASP marker that uniquely identified the resistant allele conferred by Soissons from all remaining seven susceptible founders. Finally, for QYr.niab-2D.1 two markers were converted to KASP, both of which were shown in the 90k SNP array data to distinguish the resistant Alchemy and Claire alleles from the remaining six susceptible founders. However, as the polymorphisms called for all three of these markers were null (unique to the resistant alleles from Alchemy and Claire) versus a SNP call (susceptible alleles from the six remaining founders), these markers assay for presence/absence variation, and are therefore dominant. 


\section{Discussion}

\section{Pst infection at the trial sites}

The spread of genetically diverse exotic Pst races to European environments and their displacement of the previously clonal Pst races has resulted in sudden shifts in wheat resistance ratings. The first of these Pst races to be detected in Europe is termed the 'Warrior' race (www.wheatrust.org), and was characterised by several notable traits, including relatively large reductions in resistance in varieties that previously carried effective long-term adult plant resistance (Sørensen et al. 2014) and high production of sexual stage spores (teliaspores) (Rodriguez-Algaba et al. 2014) indicative of evolution from a sexual population. In 2015, a second genetically diverse Pst pathotype called was detected for the first time in the UK, termed the 'Kranich' race (UKCPVS 2016). This race is broadly related to the 'Warrior' group had been previously detected in continental European countries, and both races are thought to have originated from sexually recombining populations in the near-Himalayan region in Asia (Hovmøller et al. 2016). Four of our trials were inoculated with a mixture of 'Solstice' and 'Warrior' Pst races, while OSG16 was naturally infected. However, natural infection was especially high in the 2015 and 2016 seasons and so likely predominated the Pst infection of all five trials. Testing of Pst isolates from the two locations used in our field trials shows these locations were dominated by natural infection by the Warrior group of races in 2015 and 2016, as was the case within the wider Cambridgeshire and Lincolnshire regions (Supplementary Table 9), and mirrored across most of the UK (UKCPVS 2016; 2017). Thus natural infection was most likely predominant in all five trials for the following reasons: (1) the high natural YR infection levels in 2015 and 2016 at these trial sites, as well as across the UK). (2) At our Lincolnshire trial sites OSG and ROTH, which were included in these surveys, the Warrior group of Pst races dominated. (3) Our own isolate testing from the NIAB15 and ROTH15 trials. (4) Our observation of natural YR infection prior to trial inoculation, and mirroring regional reports of very high YR disease pressure starting from autumn 2015 when mild conditions allowed rapid early spread (UKCPVS 2017). Therefore, we conclude that the adult plant resistance QTL identified in the MAGIC population most likely conferred resistance at the adult plant stage to the genetically diverse Pst races that characterise the recent rapid shifts in the population type of this pathogen. 
The adult plant YR resistance loci identified controlled a large percentage of the phenotypic variance, predominantly accounted for by four loci on chromosomes 1A, 2A, 2B and 2D. For all but the chromosome 1A QTL, we were able to develop KASP markers that uniquely identify all MAGIC founders conferring the resistant alleles from those conferring susceptible alleles. These resources will allow these strong-effect adult plant resistance QTL to be stacked in common genetic backgrounds. Our finding that in almost all cases, combining two or more of these QTL resulted in increased resistance provide experimental evidence that such stacking should be effective in providing strong genetic control for YR. While very good resistance was generally provided by stacking two QTL, in practice the use of more loci would provide increased security against future partial or full break-down of any single adult plant resistance locus. While there was no evidence for any of the 'major' QTL breaking down over the two seasons investigated, subsequent reports indicate that the resistance conferred by QYr.niab$2 D .1$ has been overcome (Simon Berry, personal communication). Gradual degradation of the adult plant YR resistance historically conferred by Claire since its release in 1999 (Powell et al. 2013) can first be traced to the period following the incursion of the 'Warrior' group of Pst races, when in 2011-2012 Claire went from having the highest resistance score of 9 down to an intermediate score of 6 (UKCPVS 2016). Thus, while Claire's YR resistance had partially broken down, it nevertheless contained sources of resistance unaffected by at least some of the new Pst races present in our 2015 and 2016 seasons, but which was further eroded by the breakdown of QYr.niab-2D.1. Interestingly, four adult plant YR resistance QTL have been identified on chromosomes $2 \mathrm{~B}, 2 \mathrm{D}$ and $7 \mathrm{~B}$ in field-grown trials of a Claire $\mathrm{X}$ Lemhi $(\mathrm{CxL})$ population grown in trials between 2003 and 2007 (Powell et al. 2013). The trials were conducted before the introduction of the Warrior type races in 2011, and Claire alleles conferred resistance at both loci. Of these, two QTL co-located with the adult plant resistance loci identified in the MAGIC population. CxL QTL QYr.niab-2B overlapped with MAGIC QTL QYr.niab-2B.1 (based on the CxL physical interval defined by markers $w P t-0950$ and wPt-9190: 685.047 - 750.121 Mbp). However, Soissons confers the most resistant allele in the MAGIC population, indicating that the underlying loci are most likely different, or that alleles with higher resistance than that conferred by Claire are present. CxL QTL QYr.niab-2D.2 colocated with our MAGIC QTL QYr.niab-2D.1 (based on CxL markers EST18a and wmc817a: 637.651 - 645.997 Mbp), with resistance alleles conferred by the MAGIC founders Claire and Alchemy. Notably, Alchemy has Claire in its pedigree [Alchemy = Clare $\mathrm{x}$ (Consort $\mathrm{x}$ Woodstock)] (Fradgley et al. 2019). Indeed, the haplotypes of Claire and Alchemy based on the 90k SNP data are identical across the QTL confidence interval (from SNP 
RFL_Contig1128_620 to BS00010685_51, 188.01 - $198.86 \mathrm{cM}$ ), indicating the resistance alleles carried by MAGIC founders Claire and Alchemy are identical by descent. The absence of the chromosome 2D and 7B CxL QTL in our MAGIC population may reflect changed virulence profiles of the genetically diverse $P s t$ races prevalent in our 2015/2016 season trials compared to the CxL trials that preceded the incursion of exotic Ptr races. Alternatively, it is possible that none of the MAGIC founders carry the susceptible allele that originated from Lemhi, which is a US variety. Interestingly, a recent study using the 'NIAB Elite MAGIC' population found a robust QTL conferring resistance to the necrotrophic fungal pathogen Parastagonospora nodorum (the causal agent of Septoria nodorum blotch, SNB) located in the genomic region as the major YR resistance QTL QYr.niab-2A.1 (Lin et al. 2020). However, comparison of the predicted allelic effects at these QTL finds the resistant alleles at the YR QTL carried by the founders Rialto and Xi19 to be associated with susceptibility for SNB. Analysis of multiple traits in the same population allows such correlations to be identified and further investigated. Indeed, as noted by Scott et al. (2020), the comparatively high genetic diversity and genetic recombination captured by multi-founder populations makes them well suited for the genetic analysis of multiple traits within a single experimental population, thus maximising the chances of identifying potential trade-offs between traits.

\section{Analysis of YR QTL physical intervals}

All QTL identified were outside of the pericentromeric regions that are characterised by low genetic recombination and encompass approximately half of the wheat genome (Figure 5). This, combined with high heritability of the phenotype and the strong contrasting phenotypic effect of the majority of the eight 'major' resistance QTL means that it could be useful to exploit the residual heterozygosity in the MAGIC RILs to create nearly isogenic lines for specific QTL for subsequent fine-mapping studies. Ultimately, combining such knowledge with a better understanding of the genes conferring YR susceptibility, such as the recently identified branched-chain amino acid aminotransferase gene TaBCAT1 that modulates amino acid metabolism (Corredor-Moreno et al. 2021), as well as gene editing (reviewed by Kumar et al. 2019), may allow future informed design and use of resistance alleles to enhance durable resistance to YR and other wheat pathogens. To date, just four YR adult plant resistance genes have been map-based cloned: $\operatorname{Yr15/YrG303/YrH52~(Klymiuk~et~al.~2018;~2020),~Yr18/Lr34~}$ (Krattinger et al. 2009), Yr36 (Fu et al. 2009), Yr46/Lr67 (Moore et al. 2015). However, Yr5 is known to confer resistance to a broad range of Pst isolates worldwide (Marchal et al. 2018). $\operatorname{Yr} 5$ is located in a region of chromosome $2 \mathrm{~B}$ known to contain numerous yellow rust resistance 
genes (Feng et al. 2015; Luo et al. 2009), including $Y r 7$ and $Y r S P$. Here we find the physical interval of our MAGIC adult plant resistance locus $Q Y r \cdot n i a b-2 B .1$ to overlap with the $\operatorname{Yr} 7$ and $\operatorname{Yr} 5 / Y r S p$ loci. While $Y r 7$ and $Y r S P$ no longer provide adequate resistance in the field, $\operatorname{Yr} 5$ continues to remain effective against a range of isolates worldwide (Marchal et al. 2018). $\mathrm{Yr} 7$ and $\operatorname{Yr} 5$ (represented in the wheat reference genome by gene models TraesCS2B01G488000 and TraesCS2B01G488600/TraesCS2B01G488700, respectively) are paralogous BED-NLR genes with $\sim 78 \%$ DNA identity across their coding regions (Marchal et al. 2018). YrSP however is a truncated allele of $\operatorname{Yr} 5$ resulting in the loss of most of the LRR coding region (Marchal et al. 2018). The resistant $\operatorname{Yr} 5$ and $\operatorname{YrSP}$ alleles are not reported to be present in European wheat, with $\mathrm{Yr} 5 / \mathrm{YrSP}$-specific molecular tests further confirming their absence in all eight MAGIC founder varieties (Marchal et al. 2018). However, previous analysis of available wheat genome sequence assemblies have shown that while the cultivars Claire and Robigus (two of our eight MAGIC founders) lack $Y r 7$, they do contain haplotypes at the gene underlying Yr5 that encode proteins that differ by 12 and 33 amino acids to the YR resistant $\operatorname{Yr} 5$ allele, respectively (Marchal et al. 2018). However, neither Claire nor Robigus were reported as showing the $\mathrm{Yr} 5 / \mathrm{YrSP}$ resistance response, indicating these changes in the protein do not affect function. In our MAGIC population, resistance at QTL QYr.niab-2B.1 came from the Soissons allele. Collectively, this indicates that while $\mathrm{Yr} 7$ and $\mathrm{Yr} 5 / \mathrm{YrSP}$ do not themselves confer resistance at QYr.niab-2B.1, it is possible that different mutations at their underlying genes, or allelic variation at the three additional paralogous BED-NLRs in the vicinity, could underly resistance at this locus. Ultimately, further investigation of these, and other, candidates at the QTL is required to narrow down the underlying causative gene and polymorphism.

\section{Conclusions}

Since 2011, several genetically diverse Pst races have spread rapidly across Europe from their centre of origins in Asia. Better understanding of the genes and gene networks underlying adult plant resistance in the face of such isolates will help breeding for YR resistance. Collectively, the genetic information, wheat germplasm and molecular markers generated here provide resource that could be used to help design and develop wheat varieties with improved adult plant genetic resistance to emerging races of yellow rust. 
584

585

586

587

588

589

590

591

592

593

594

595

596

597

598

599

600

601

602

603

604

605

606

607

608

609

610

611

612

613

614

615

616

\section{Declarations}

Funding: LB was funded via a Biotechnology and Biological Sciences Research Council (BBSRC) Doctoral Training Partnership PhD award to the University of Cambridge. LB also received funding from the Roger Harrison Trust towards travel expenses. JC's time was partly supported by BBSRC grant BB/N00518X/1, funded via the $2^{\text {nd }}$ call of the ERA-CAPs framework.

Conflicts of interest: the authors declare no conflict of interests.

Availability of data and material: Phenotypic data is available in the Supplementary Materials. Germplasm for the 'NIAB Elite MAGIC' population is available via https://www.niab.com/research/agricultural-crop-research/resources.

Code availability: not applicable.

Author contributions: LB carried out trial preparation, pathology work, yellow rust assessments and all statistical and genetic analyses. SB and PW managed the trials at Osgodby and Rothwell. LB, CM, LP and JC undertook bioinformatics. LB and JC developed KASP markers. IM, SH and JC provided project supervision and resources. LB and JC wrote the manuscript. All authors edited and approved the manuscript.

Ethics approval: not applicable.

Consent to participate: not applicable.

Consent for publication: all authors give consent.

Acknowledgements: We thank Prof Julian Hibberd (University of Cambridge) for supervisory inputs within the Doctoral Training Programme, Keith Gardner (NIAB) for quantitative genetics advice, and the NIAB Trials Team for the sowing, growth and agronomic care of the NIAB field trails. We also thank Marie Gantet, Trynstje Broesma, Aurélie Jouanin and Doriane Dam for their support with yellow rust assessments. 


\section{References}

618

Akaike H (1974) A new look at the statistical model identification. IEEE Trans Automat Contr 620 19:716-723

621

622 Alaux M, Rogers J, Letellier T, Flores R, Alfama F, Pommier C, Mohellibi N, Durand S, 623 Kimmel E, Michotey C, Guerche C, Loaec M, Lainé M, Steinbach D, Choulet F, Rimbert H, 624 Leroy P, Guilhot N, Salse J, Feuillet C; International Wheat Genome Sequencing Consortium, 625 Paux E, Eversole K, Adam-Blondon AF, Quesneville H (2018) Linking the International 626 Wheat Genome Sequencing Consortium bread wheat reference genome sequence to wheat genetic and phenomic data. Genome Biol 19:111.

628

Ali S, Gladieux P, Leconte M, Gautier A, Justesen AF, et al. (2014) Origin, migration routes and worldwide population genetic structure of the wheat yellow rust pathogen Puccinia striiformis f.sp. tritici. PLoS Pathol 10:e1003903.

632

Ali S, Rodriguez-Algaba J, Thach T, Sørensen CK, Hansen JG, Lassen P, Nazari K, Hodson DP, Justesen AF, Hovmøller MS (2017) Yellow rust epidemics worldwide were caused by pathogen races from divergent genetic lineages. Front Plant Sci 8:1057.

Altschul SF, Gish W, Miller W, Myers EW, Lipman DJ (1990) Basic local alignment search 638 tool. J Mol Biol 215:403-410.

639

640

Andersen EJ, Nepal MP, Purintun JM, Nelson D, Mermigka G, Sarris PF (2020) Wheat disease resistance genes and their diversification through integrated domain fusions. Front Genet 11:898.

644 Andersson MX, Kourtchenko O, Dangl JL, Mackey D, Ellerström M (2006) Phospholipasedependent signalling during the AvrRpm1- and AvrRpt2-induced disease resistance responses in Arabidopsis thaliana. Plant J 47:947-59. cloning from a wild crop relative by sequence capture and association genetics. Nature Biotechnol 37:139-143. 
Axtell MJ, Chisholm ST, Dahlbeck D, Staskawicz BJ (2003) Genetic and molecular evidence that the Pseudomonas syringae type III effector protein AvrRpt2 is a cysteine protease. Mol Microbiol 49:1537-1546 .

655

Bates D, Mächler M, Bolker B, Walker S (2015) Fitting linear mixed-effects models using lme4. J Stat Softw 67:1-48. doi: 10.18637/jss.v067.i01.

658

659

Bayles R, Flath K, Hovmøller M, de Vallavieille-Pope C (2000) Breakdown of the $\operatorname{Yr} 17$ 660 resistance to yellow rust of wheat in northern Europe. Agronomie 20:805-811.

661

662

Brueggeman R, Rostoks N, Kudrna D, Kilian A, Han F, Chen J, Druka A, Steffenson B, 663 Kleinhofs A (2002) The barley stem rust-resistance gene Rpgl is a novel disease-resistance 664 gene with homology to receptor kinases. Proc Natl Acad Sci USA 99:9328-9333.

665

Bueno-Sancho V, Persoons A, Hubbard A, Cabrera-Quio LE, Lewis CM, Corredor-Moreno P, 667 Bunting DCE, Ali S, Chng S, Hodson DP, Burrows RM, Bryson R, Thomas J, Holdgate S, 668 Saunders DGO (2017) Pathogenomic analysis of wheat yellow rust lineages detects seasonal 669 variation and host specificity. Genome Biol Evol 9:3282-3296.

Chen W, Wellings C, Chen X, Kang Z, Liu T (2014) Wheat stripe (yellow) rust caused by Puccinia striiformis f sp. tritici. Mol Plant Pathol 15:433-446.

673

Cockram J, Mackay I (2018) Genetic mapping populations for conducting high-resolution trait mapping in plants. In: Varshney R, Pandey M, Chitikineni A (eds) Plant genetics and molecular biology. Advances in Biochemical Engineering/Biotechnology, vol 164. Springer, Cham.

678 Corredor-Moreno P, Minter F, Davey PE, Wegel E, Kular B, Brett P, Lewis CM, Morgan YML, Macías Pérez A, Lorolev AV, Hill L, Saunders DGO (2021) The branched-chain amino acid aminotransferase TaBCAT1 modulates amino acid metabolism and positively regulates wheat rust susceptibility. Plant Cell https://doi.org/10.1093/plcell/koab049. 
685

686

687

688

689

690

691

692

693

694

695

696

697

698

699

700

701

702

703

704

705

706

707

708

709

710

711

712

713

714

715

716

717

718

wheat sensitivity to the ToxB fungal effector from Pyrenophora tritici-repentis, the causal agent of Tan Spot. Theor Appl Genet 133:939-950.

Downie RC, Bouvet L, Furuki E, Gosman N, Gardner KA, Mackay IJ, Mantello CC, Mellers G, Phan HTT, Rose GA, Tan K-C, Oliver RP, Cockram J (2018) Assessing European wheat sensitivities to Parastagonospora nodorum necrotrophic effectors and fine-mapping of the Snn3-B1 locus conferring sensitivity to the effector SnTox3. Front Plant Sci 9:881.

Downie RC, Lin M, Borsi B, Ficke A, Lillemo M, Oliver RP, Phan H, Tan K-C, Cockram J (2020) Spetoria nodorum blotch of wheat: disease management and resistance breeding in the face of shifting disease dynamics and a changing environment. Phytopathol doi: https://doi.org/10.1094/PHYTO-07-20-0280-RVW.

Edmondson RN (2020) Multi-level block designs for comparative experiments. J Agr Biol Envir St 25:500-522.

Fragley N, Gardner KA, Cockram J, Elderfield J, Hickey JA, Howell P, et al. (2019) A largescale pedigree resource of wheat reveals evidence for adaptation and selection by breeders. PLOS Biol 17:e3000071.

Fu D, Uauy C, Distelfeld A, Bechl A, Epstein L, Chen X, Sela H, Fahima T, Dubcovsky J (2009) A novel kinase-START gene confers temperature-dependent resistance to wheat stripe rust. Science 322:1357-1360.

Gardner KA, Wittern LM, Mackay IJ (2016) A highly recombined, high-density, eight founder wheat MAGIC map reveals extensive segregation distortion and genomic locations of introgression segments. Plant Biotechnol J 14:1406-1417.

He H, Zhu S, Zhao R, Jiang Z, Ji Y, Ji J, Qiu D, Li H, Bie T (2018) Pm21, encoding a typical CC-NBS-LRR protein, confers broad-spectrum resistance to wheat powdery mildew disease. Mol Plant 11:879-882.

Huang BE, George AW (2011) R/mpMap: a computational platform for the genetic analysis of multiparent recombinant inbred lines. Bioinformatics 27:727-729. 
Hubbard A, Lewis C, Yoshida K, Ramirez-Gonzalez R, de Vallavieille-Pope C, Thomas J,

721

Kamoun S, Bayles R, Uauy C, Saunders DG (2015) Field pathogenomics reveals the emergence of a diverse wheat yellow rust population. Genome Biol 16:23.

Hurni S, Brunner S, Buchmann G, Herren G, Jordan T, Krukowski P, Wicker T, Yahiaoui Nm Mago R, Keller B (2013) Rye Pm8 and wheat Pm3 are orthologous genes and show evolutionary conservation of resistance function against powdery mildew. Plant J 76:957-969.

Hovmøller MS, Walter S, Bayles RA, Hubbard A, Flath K, Sommerfeldt N, Leconte M, Czembor P, Rodriguez-Algaba J, Thach T, Hansen JG, Lassen P, Justesen AF, Ali S, de Vallavieille-Pope C (2016) Replacement of the European wheat yellow rust population by new races from the centre of diversity in the near-Himalayan region. Plant Pathol 65:402-411.

Hubbard A, Lewis C, Yoshida K, Ramirez-Gonzalez R, de Vallavieille-Pope C, Thomas J. et al. (2015) Field pathogenomics reveals the emergence of a diverse wheat yellow rust population. Genome Biol 16:23.

The International Wheat Genome Sequencing Consortium (IWGSC), Appels R, Eversole K, Stein N, Feuillet C, Keller B, Rogers J Pozniak C, et al. (2018) Shifting the limits in wheat research and breeding using a fully annotated reference genome. Science 361:eaar7191.

Jamil S, Shahzad R, Ahmad S, Fatima R, Zahid R, Anwar M, Iqbal MZ, Wang X (2020) Role of genetics, genomics and breeding approaches to combat stripe rust of wheat. Front Nutr 7:580715.

Jones JD, Vance RE, Dangl JL (2016) Intracellular innate immune surveillance devices in plants and animals. Science 354:aaf6395.

Kertho A, Mamidi S, Bonman JM, McClean PE, Acevedo M (2015) Genome-wide association mapping for resistance to leaf and stripe rust in winter-habit hexaploid wheat landraces. PLoS ONE 10:e0129580. 
752

753

754

755

756

757

758

759

760

761

762

763

764

765

766

767

768

769

770

771

772

773

774

775

776

777

778

779

780

781

782

783

784

785

Klymiuk V, Taniv E, Huang L, Raats D, Fatiukha A, et al. (2018) Cloning of the wheat Yr15 resistance gene sheds light on the plant tandem kinase-pseudokinase family. Nat Commun 9:3735.

Klymiuk V, Fatiukha A, Raats D, Bocharova V, Huang L, Feng L, Jaiwar S, Pozniak Cm Coaker G, Dubcovsky J, Fahima T (2020) Three previously characterized resistances to yellow rust are encoded by a single locus Wtk1. J Exp Bot 71:2561-2572.

Krzywinski M, Schein J, Birol I, Connors J, Gascoyne R, Horsman D, Jones SJ, Marra MA (2009) Circos: an information aesthetic for comparative genomics. Genome Res 19:1639-45.

Kumar R, Kaur A, Pandey A, Mamrutha HM, Singh GP (2019) CRISPR-based genome editing in wheat: a comprehensive review and future prospects. Mol Biol Rep 46:3557-3569.

Li M, Dong L, Li B, Wang Z, Xie J, et al. (2020) A CNL protein in wild emmer wheat confers powdery mildew resistance. New Phytol 228:1027-1037.

Lin M, Corsi B, Ficke A, Tan K-C, Cockram J, Lillemo M (2020) Genetic mapping using a wheat multi-founder population reveals a locus on chromosome $2 \mathrm{~A}$ controlling resistance to both leaf and glume blotch caused by the necrotrophic fungal pathogen Parastagonospora nodorum. Theor Appl Genet 133:785-808.

Loutre C, Wicker T, Travella S, Galli P, Scofield S, Fahima T, Feuillet C, Keller B (2009) Two different CC-NBS-LRR genes are required for $\mathrm{Lr} 10$-mediated leaf rust resistance in tetraploid and hexaploid wheat. Plant J 60:1043-1054.

Luo PG, Hu XY, Ren ZL, Zhang HY, Shu K, Yang ZJ (2008) Allelic analysis of stripe rust resistance genes on wheat chromosome 2BS. Genome 51:922-927.

Mackay TF (2001) The genetic architecture of quantitative traits. Annu Rev Genet 35:303339.

Maccaferri M, Zhang J, Bulli P, Abate Z, Chao S, Cantu D, Bossolini E, Chen X, Pumphrey M, Dubcovsky J (2015) A genome-wide association study of resistance to stripe rust (Puccinia 
786

787

788

789

790

791

792

793

794

795

796

797

798

799

800

801

802

803

804

805

806

807

808

809

810

811

812

813

814

815

816

817

striiformis f. sp. tritici) in a worldwide collection of hexaploid spring wheat (Triticum aestivum L.). G3: Genes, Genomes, Genet 5:449-465.

Mackay IJ, Bansept-Basler P, Barber T, Bentley AR, Cockram J, Gosman N, Greenland AJ, Horsnell R, Howells R, O’Sullivan DM, Rose GM, Howell PH (2014) An eight-parent multiparent advanced generation inter-cross population for winter sown wheat: creation, properties, and validation. G3: Genes Genomes Genet 4:1603-1610.

Mackey D, Holt $3^{\text {rd }}$ BF, Wiig A, Dangl JL (2002) RIN4 interacts with Pseudomonas syringae type III effector molecules and is required for RPM1-mediated resistance in Arabidopsis. Cell 108:743-54.

Marchal C, Zhang J, Zhang P, Fenwick P, Steuernagel B, Adamski NM, Boyd L, McIntosh R, Wulff BBH, Berry S, Lagudah E, Uauy C (2018) BED-domain-containing immune receptors confer diverse resistance spectra to yellow rust. Nature Plants 4:662-668.

Milus EA, Kristensen K, Hovmøller MS (2009) Evidence for increased aggressiveness in a recent widespread strain of Puccina striiformis f. sp. tritici causing stripe rust of wheat. Phytopathol 99:89-94.

Peterson RF, Campbell AB, Hannah AE (1948) A diagrammatic scale for estimating rust intensity on leaves and stems of cereals. Can J Res 26c:496-500.

Powell NM, Lewis CM, Berry ST, MacCormack R, Boyd LA (2013) Stripe rust resistance genes in the UK winter wheat cultivar Claire. Theor Appl Genet 126:1599-1612.

R Core Team (2013) R: A language and environment for statistical computing. R Foundation for Statistical Computing, Vienna, Austria. URL http://www.R-project.org/.

Ramirez-Gonzalez R, Uauy C, Caccamo M (2015) PolyMarker: A fast polyploid primer design pipeline. Bioinformatics 31:2038-2039. 
818

819

820

821

822

823

824

825

826

827

828

829

830

831

832

833

834

835

836

837

838

839

840

841

842

843

844

845

846

847

848

849

Rodriguez-Algaba J, Walter S, Sørensen CK, Hovmøller MS, Justesen AF (2014) Sexual structures and recombination of the wheat rust fungus Puccinia striiformis on Berberis vulgaris. Fungal Genet Biol 70:77-85.

Rosewarne GM, Herrera-Foessel SA, Singh RP, Huerta-Espino J, Lan CX, He ZH (2013)

Quantitative trait loci of stripe rust resistance in wheat. Theor Appl Genet 126:2427-2449.

Sánchez-Martin J, Steuernagel B, Ghosh S, Herren G, Hyrni S, et al. (2016) Rapid gene isolation in barley and wheat by mutant chromosome sequencing. Genome Biol 17:221.

Sarris P, Cevik V, Dagadas G, Jones JDG, Krasileva KV (2016) Comparative analysis of plant immune receptor architectures uncovers host proteins likely targeted by pathogens. BMC Biol $14: 8$.

Scott M, Ladejobi O, Sammer A, Bentely AR, Biernaskie J, Bowden SA, Dell'Acqua M, Dixon LE, Filippi CV, Fradgley N, Gardner KA, Mackay IJ, O'Sullivan D, Percival-Alwyn L, Roorkiwal M, Singh RK, Thudi M, Varshney RK, Venturini L, Whan A, Cockram J, Mott R (2020) Multi-parent populations in crops: a toolbox integrating genomics and genetic mapping with breeding. Heredity 125:396-416.

Singh RP, Huerta-Espino J, William HM (2005) Genetics and breeding for durable resistance to leaf and stripe rusts in wheat. In: Turkish J Agric Forestry 29:121-127.

Sørensen CK. Hovmøller MS, Leconte M, Dedryver F, de Vallavieille-Pope C (2014) New races of Puccina striiformis found in Europe reveal race specificity of long-term effective adult plant resistance in wheat. Phytotaphol 104:1042-1051.

Steuernagel B, Witek K, Krattinger SG, Ramirez-Gonzalez RH, Schoonbeek J-J, Yu G, Baggs E, Witek AI, Yadav I, Krasileva KV, Jones JDG, Uauy C, Keller B, Ridout CJ, Wulff BBH (2020) The NLR-annotator tool enables annotation of the intracellular immune receptor repertoire. Plant Physiol https://doi.org/10.1104/pp.19.01273. 
850

851

852

853

854

855

856

857

858

859

860

861

862

863

864

865

866

867

868

869

870

871

872

873

874

875

876

877

878

879

880

881

882

Thind AK, Wicker T, Šimková H, Fossati D, Moullet O, Brabant C, Vrána J, Doležel J, Krattinger SG (2017) Rapid cloning of genes in hexaploid wheat using cultivar-specific longrange chromosome assembly. Nat Biotechnol 35:793-796.

UKCPVS (2016) United Kingdom cereal pathogen virulence survey 2016 annual report. https://ahdb.org.uk/ukcpvs .

VSN International (2015) Genstat for Windows. VSN International, Hemel Hempstead, $18^{\text {th }}$ Edition. UK webpage: www.genstat.co.uk.

Wang M, Chen X (2017) Stripe Rust Resistance. In: Chen X, Kang Z. (eds) Stripe rust. Springer, Dordrecht.

Wang S, Wong D, Forrest K, Allen A, Chao S, Huang BE, et al. (2014) Characterization of polyploid wheat genomic diversity using a high-density 90,000 single nucleotide polymorphism array. Plant Biotechnol J 12:787-796.

Wellings CR (2011) Global status of stripe rust: a review of historical and current threats. Euphytica 179:129-141.

Wilcoxon RD, Skovmand B, Atif AH (1975) Evaluation of wheat cultivars for ability to retard development of stem rust. Ann Appl Biol 80:275-281.

Zadoks Z, Chang TT, Konzak CF (1974) A decimal code for the growth stages of cereals. Weed Res 14:415-421.

Zegeye H, Rasheed A, Makdis F, Badebo A, Ogbonnaya FC (2014) Genome-wide association mapping for seedling and adult plant resistance to stripe rust in synthetic hexaploid wheat. PLoS ONE 9:e105593.

Zhang W, Chen S, Abate Z, Nirmala J, Rouse MN, Dubcovsky J (2017) Identification and characterization of $\mathrm{Sr} 13$, a tetraploid wheat gene that confers resistance to the Ug99 stem rust race group. Proc Natl Acad Sci U S A 114:E9483-E9492. 


\section{Figure legends}

Figure 1. Examples of yellow rust resistance phenotypes in the MAGIC population. Assessments were made using the modified Cobb's scale (Peterson et al. 1948) ranging from 0 to $100 \%$ : (a) score=0, no infection, 0\%, (b) some tillers infected with a few stripes 0 - $15 \%$, (c) most tillers infected with several stripes, 15-25\%, (d) all leaves infected, 25-50\%, (e) all leaves infected, 50-75\%, (f) little to no green tissue left, $>75 \%$.

Figure 2. Yellow rust resistance in the MAIGC population. Five test environments: NIAB, Osgodby and Rothwell in 2015 (NIAB15, OSG15, ROTH15) and at NIAB and Osgodby in 2016 (NIAB16, OSG16). At each site, YR infection was recorded at two timepoints: S1 (score 1) and S2 ( score 2). $\mathrm{Al}=$ Alchemy, $\mathrm{Br}=$ Brompton, $\mathrm{Cl}=$ Claire, $\mathrm{He}=$ Hereward, $\mathrm{Ri}=$ Rialto, $\mathrm{Ro}=$ Robigus, So $=$ Soissons, $\mathrm{Xi}=\mathrm{Xi19} .(\mathrm{A}) \mathrm{MAGIC}$ founder percentage YR resistance scores. Additionally, boxplots for all MAGIC recombinant inbred lines are indicated for each trial. (B) Histograms of percentage YR infection in the MAGIC recombinant inbred lines (RILs). Data for the non-transformed adjusted means (BLUEs) are shown for the RILs,. BLUEs for each of the eight founders are overlaid below, following the colour coding shown in the key. Note: for QTL analysis, the 2016 season data was log transformed. Negative values $=0$. Values over $100=100$.

Figure 3. Examples of yellow rust resistance genetic analysis. Shown is the NIAB 15 dataset analysed using composite interval mapping with 10 covariates (CIM-cov10), showing QTL scans using the following phenotypes: (A) scoring time-point 1, (B) scoring time-point 1, and (C) scoring time-point 2. All 21 wheat chromosomes are shown, with $1=$ chromosome $1 \mathrm{~A}, 2=1 \mathrm{~B}, 3=1 \mathrm{D}$, through to $21=7 \mathrm{D}$.

Figure 4. The effects of combining yellow rust (YR) resistance alleles at multiple QTL. Boxplots of the distribution of YR percentage infection in MAGIC recombinant inbred lines (RILs) in each of the five field trials. Based on the presence of resistance alleles at up to four of the most significant MAGIC YR resistance quantitative trait loci (QTL): on chromosomes 1A (QYr.niab-1A.1), 2A (QYr.niab-2A.1), 2B (QYr.niab-2B.1) 
and 2D (QYr.niab-2D.1). Boxes denote the interquartile range, the horizontal lines that bisect them the median, the vertical lines that extend from them the $1.5 \mathrm{x}$ interquartile range beyond it, and the dots the outlier MAGIC RILs.

Figure 5. Locations of the eight most significant yellow rust QTL visualised on plots of all genetically mapped SNPs in the MAGIC genetic map (cM) (Gardner et al. 2016) versus their physical map locations (Mbp) (IWGSC Refseq v1.0). Shown are: (A) QYr.niab-1A.1 (represented by peak SNP RAC875_rep_c71093_1070) on chromosome 1A, (B) QYr.niab-2A.1 (BS00022903_51) and QYr.niab-2A.2 (BS00011599_51) on chromosome 2A. (C) QYr.niab-2B.1(Kukri_c9118_1774) on chromosome 2B. (D) QYr.niab-2D.1 (Ra_c21099_1781) on chromosome 2D. (E) QYr.niab-3A.1 (Kukri_c28650_111) on chromosome 3A. (F) QYr.niab-6A.1 (BS00011010_51) and QYr.niab-6A.2 (Kukri_c21743_269) on chromosome 6A.

Figure 6. Circular diagram representing the position of 14 yellow rust (YR) resistance QTL identified in the MAGIC wheat population, in relation to density of candidate 'resistance genes' present in the cv. Chinese Spring reference genome (RefSeq v1.0; IWGSC, 2018). Starting from the outermost, Track 1: chromosome number. Track 2: tick marks indicating the position of major (red) and minor (green) YR QTL. Track 3 (purple): histogram of the proportion of resistant genes per gene per 10Mbp. Track 4 (blue): histogram of 'resistant gene' count every $10 \mathrm{Mbp}$. Track 5 (dark blue): histogram of gene count every $10 \mathrm{Mbp}$.

Figure 7. Examples of genotyping results for some of the KASP markers developed for the four most significant yellow rust resistance QTL (see Supplementary Table 2 for full details). Genotype calls for resistant (Res) and susceptible (Sus) alleles are indicated, derived from genomic DNA template from the founders subsequently indicated here. NTC = no template negative control. (A) QYr.niab-1A.1: KASP marker tplb0021i12_383 (DNA: Alchemy, susceptible allele; Hereward, resistant allele). (B) QYr.niab-2A.1: KASP marker BS00062679_51 (DNA: Xi19, resistant allele; Claire, susceptible allele). (C) QYr.niab-2B.1: KASP marker BS00016650_51 (DNA: Soissons, resistant allele; Robigus, susceptible allele). (D) QYr.niab-2D.1: KASP marker Kukri_c498_2381 (DNA: Claire, resistant allele; Soissons, susceptible allele). 


\begin{tabular}{|c|c|c|c|c|c|}
\hline Trial & NIAB15 & OSG15 & ROTH15 & NIAB16 & OSG16 \\
\hline Location & Cambridge & Osgodby & Rothwell & Cambridge & Osgodby \\
\hline Total number of plots & 2,208 & 1,200 & 1,200 & 1,200 & 1,200 \\
\hline Columns $\mathrm{x}$ rows & 48 cols $x 46$ rows & 20 cols $\times 60$ rows & 20 cols $\times 60$ rows & 20 cols $\times 60$ rows & 20 cols x 60 rows \\
\hline Main : blocks : subblocks per block & $2: 46: 0$ & - & - & $2: 3: 2$ & $2: 3: 2$ \\
\hline MAGIC RILs replicated & 1,085 & - & - & 444 & 444 \\
\hline MAGIC RILs unreplicated & - & 1,060 & 1,060 & 234 & 234 \\
\hline MAGIC founders & 8 & 8 & 8 & $8^{\dagger}$ & $8^{\dagger}$ \\
\hline Varietal controls & $10^{\mathrm{a}}$ & $2^{\mathrm{b}}$ & $2^{\mathrm{b}}$ & $2^{\dagger}$ & $2^{\dagger}$ \\
\hline MAGIC founder controls & - & $20^{c}$ & $20^{\mathrm{c}}$ & - & - \\
\hline Additional checks & - & Vuka $a^{\mathfrak{L}}$ & Vuka ${ }^{\mathfrak{f}}$ & - & - \\
\hline
\end{tabular}

Table 1. Overview of the five yellow rust MAGIC trials undertaken in the United Kingdom during the 2015 and 2016 seasons. An unbalanced incomplete random block design was used for all trials. ${ }^{a}$ Controls: the yellow rust differential lines Ambition (YR resistance gene $\left.A m\right)$, Cadenza $(\mathrm{Ca})$, KWS Sterling $(\mathrm{St})$, Rendezvous (Re), Solstice (So), Spaldings Prolific ( $\mathrm{Sp}$ ), Timber ( $\mathrm{Ti})$, Warrior $(\mathrm{Wa})$, the positive control Vuka (-) and the negative control Cougar $(\mathrm{Co}) .{ }^{\mathrm{b}}$ Controls: Vuka (positive control) and Cougar (negative control). ${ }^{\mathrm{c}} 20 \mathrm{MAGIC}$ RIL lines used for controls, listed in Supplementary Table 1). All lines and controls were present as two replicates, unless otherwise stated: ${ }^{\dagger}$ Control varieties Oakley (positive control) and Cougar (negative control), each replicated three times. ${ }^{\mathfrak{f}}$ Susceptible variety Vuka replicated 80 times. 


\begin{tabular}{|c|c|c|c|c|c|c|c|c|c|c|c|}
\hline \multirow[t]{2}{*}{ QTL } & \multirow[t]{3}{*}{ Chr. } & \multicolumn{10}{|c|}{ Environments } \\
\hline & & \multicolumn{2}{|c|}{ NIAB15 } & \multicolumn{2}{|c|}{ OSG15 } & \multicolumn{2}{|c|}{ ROTH15 } & \multicolumn{2}{|c|}{ NIAB16 } & \multicolumn{2}{|c|}{ OSG16 } \\
\hline $\mathbf{A}$ & & S1 & S2 & S1 & S2 & S1 & S2 & S1 & S2 & S1 & $\mathrm{S} 2$ \\
\hline QYr.niab-1A.1 & $1 \mathrm{~A}$ & $1-4$ & $1-4$ & $1-4$ & $1-4$ & $1-2$ & $1-2$ & $1-4$ & $1-4$ & $1-4$ & $1-4$ \\
\hline QYr.niab-2A.1 & $2 \mathrm{~A}$ & $1-4$ & $1-4$ & $1-4$ & $1-4$ & $1-4$ & $1-4$ & $1-4$ & $1-4$ & $1-4$ & $1-4$ \\
\hline QYr.niab-2A.2 & $2 \mathrm{~A}$ & $1-2$ & $1-2$ & $1-2$ & $1-2$ & $1-2$ & 1 & $1-2$ & $1-2$ & 1 & 1 \\
\hline QYr.niab-2B.1 & $2 \mathrm{~B}$ & $1-4$ & $1-4$ & $1-4$ & $1-4$ & $1-4$ & $1-4$ & $1-4$ & $1-4$ & $1-4$ & $1-4$ \\
\hline QYr.niab-2D.1 & $2 \mathrm{D}$ & $1-4$ & $1-4$ & $1-4$ & $1-4$ & $1-4$ & $1-4$ & $1-4$ & $1-4$ & $1-4$ & $1-4$ \\
\hline QYr.niab-3A.1 & $3 \mathrm{~A}$ & $1-4$ & $1-4$ & $1-4$ & $1-4$ & $1-2$ & $1-2$ & $2-4$ & 3 & $1-4$ & $1-4$ \\
\hline QYr.niab-6A.1 & $6 \mathrm{~A}$ & $2-4$ & $2-4$ & $3-4$ & $2-4$ & 2 & 2 & $2-4$ & $1-4$ & $1-4$ & $1-4$ \\
\hline QYr.niab-6A.2 & $6 \mathrm{~A}$ & $1-2$ & $1-2$ & $1-2$ & $1-2$ & $1-2$ & $1-2$ & $1-2$ & $1-2$ & $1-2$ & $1-2$ \\
\hline B & & & & & & & & & & & \\
\hline QYr.niab-3D.1 & $3 \mathrm{D}$ & 2 & 2 & $1-2$ & $1,2,4$ & $1-3$ & $1-3$ & 2 & 2 & & \\
\hline QYr.niab-4B.1 & $4 \mathrm{~B}$ & & & & & & & $1-4$ & & $1-2$ & \\
\hline QYr.niab-4D.1 & $4 \mathrm{D}$ & $1-2$ & 2 & & & & & & & $1-4$ & $1-3$ \\
\hline QYr.niab-5A.1 & $5 \mathrm{~A}$ & $1,2,4$ & $1,2,4$ & & & 1 & & & 1 & & \\
\hline QYr.niab-6A.3 & $6 \mathrm{~A}$ & 2 & & 2 & 2 & 2 & 2 & 2 & 2 & $1-2$ & $1-2$ \\
\hline QYr.niab-6B.1 & $6 \mathrm{~B}$ & & 4 & $1-4$ & $1-4$ & $2-3$ & $2-3$ & & & & \\
\hline
\end{tabular}

Table 2. 'Major' (A) and 'minor' (B) yellow rust resistance QTL identified in the MAGIC population using four different QTL analysis methods. QTL analysis methods are numbered as: $1=$ single marker analysis, $2=$ identity by descent (IBD), $3=$ interval mapping, $4=$ composite interval mapping with 10 covariates. Trial sites: NIAB, Osgodby (OSG), Rothwell (ROTH). Trial years: 2015 and 2016. Yellow rust resistance phenotyping was undertaken at two timepoints: score-1 (S1) and score-2 (S2). Chr. = chromosome. QTL were detected at $5 \%$ significance threshold. 


\begin{tabular}{|c|c|c|c|c|c|c|c|c|c|}
\hline QTL & Chr. & Peak SNP marker & $\begin{array}{l}\text { Genetic } \\
\text { position } \\
(\mathbf{c M}) \\
\end{array}$ & $\begin{array}{l}\text { Physical } \\
\text { position } \\
\text { (Mbp) }\end{array}$ & $p$-value & & $\begin{array}{l}\% \text { var } \\
\text { explai }\end{array}$ & ned & $\begin{array}{l}\text { QTL } \\
\text { interval } \\
\text { (cM) }\end{array}$ \\
\hline & & & & & $\min$ & $\max$ & $\min$ & $\max$ & \\
\hline QYr.niab-1A.1 & $1 \mathrm{~A}$ & RAC875_rep_c71093_1070 & 185.80 & 568.013 & $5.72 \mathrm{E}-10$ & $1.23 \mathrm{E}-05$ & 7.46 & 9.26 & 17.17 \\
\hline QYr.niab-2A.1 & $2 \mathrm{~A}$ & BSO0022903_51 & 140.26 & 607.827 & $3.07 \mathrm{E}-13$ & $1.24 \mathrm{E}-08$ & 6.80 & 9.37 & 19.29 \\
\hline QYr.niab-2A.2 & $2 \mathrm{~A}$ & BS00011599_51 & 259.39 & 762.290 & $1.75 \mathrm{E}-08$ & 7.91E-03 & - & - & 2.56 \\
\hline$\overline{Q Y r . n i a b-2 B .1}$ & $2 \mathrm{~B}$ & Kukri_c9118_1774 & 271.92 & 683.048 & $0^{*}$ & $1.58 \mathrm{E}-11$ & 7.38 & 17.72 & 18.62 \\
\hline QYr.niab-2D.1 & $2 \mathrm{D}$ & Ra_c21099_1781 & 197.36 & 638.376 & $0^{*}$ & $8.62 \mathrm{E}-12$ & 10.83 & 18.38 & 10.85 \\
\hline$\overline{Q Y r . n i a b-3 A .1}$ & $3 \mathrm{~A}$ & Kukri_c28650_111 & 3.02 & 7.921 & $1.41 \mathrm{E}-07$ & $5.58 \mathrm{E}-04$ & 3.48 & 6.02 & 16.64 \\
\hline QYr.niab-3D.1 & $3 \mathrm{D}$ & BS00004334_51 & 162.20 & 574.773 & $3.30 \mathrm{E}-05$ & $1.40 \mathrm{E}-03$ & 3.35 & - & 0 \\
\hline QYr.niab-4B.1 & $4 \mathrm{~B}$ & $R a \_c 26080 \_461$ & 50.66 & 36.643 & $3.72 \mathrm{E}-05$ & - & - & 2.97 & 17.73 \\
\hline QYr.niab-4D.1 & $4 \mathrm{D}$ & D_GDRF1KQ02H66WD_341 & 125.78 & 499.107 & 1.-7E-05 & $2.44 \mathrm{E}-04$ & 2.89 & 4.03 & 26.56 \\
\hline QYr.niab-5A.1 & $5 \mathrm{~A}$ & $I A A V 3916$ & 301.25 & 683.343 & $1.21 \mathrm{E}-04$ & $9.62 \mathrm{E}-03$ & 3.48 & 3.60 & 13.32 \\
\hline QYr.niab-6A.1 & $6 \mathrm{~A}$ & BS00011010_51 & 55.51 & 18.713 & $4.52 \mathrm{E}-10$ & $1.22 \mathrm{E}-05$ & 5.50 & 6.57 & 18.24 \\
\hline QYr.niab-6A.2 & $6 \mathrm{~A}$ & Kukri_c21743_269 & 75.69 & 27.108 & $5.15 \mathrm{E}-10$ & $9.84 \mathrm{E}-05$ & - & - & 2.05 \\
\hline$\overline{Q Y r . n i a b-6 A .3}$ & $6 \mathrm{~A}$ & wsnp_Ex_rep_c101766_87073440 & 220.32 & 596.521 & $1.49 \mathrm{E}-04$ & $1.37 \mathrm{E}-04$ & - & - & 17.96 \\
\hline QYr.niab-6B.1 & $6 \mathrm{~B}$ & BSO0068615_51 & 60.56 & 54.662 & $1.44 \mathrm{E}-07$ & $1.44 \mathrm{E}-05$ & 2.49 & 4.08 & 13.37 \\
\hline
\end{tabular}

Table 3 Details of the 14 yellow rust (YR) resistance QTL identified in the MAGIC population in seasons 2015 and 2016. The nine 'major' QTL are highlighted in bold. Pairs of QTL located in broadly colinear positions on homoeologous chromosomes are underlined. Chr. = chromosome. Genetic map position and QTL interval are from the MAGIC genetic map (Gardner et al. 2016). Minimum (min) and maximum (max) values for $p$ value and percentage phenotypic variance explained (\% var explained) selected amongst all environments and QTL mapping methods. ${ }^{*}<2.22 \mathrm{E}^{-}$ 16. QTL interval based on QTL mapping method-4 (composite interval mapping, 10 covariates). 


\begin{tabular}{|c|c|c|c|c|c|c|c|c|c|c|}
\hline QTL & Chr & Predic & d foun & er effect & & & & & & Origin \\
\hline & & Al & $\mathrm{Br}$ & Cl & He & $\mathbf{R i}$ & Ro & So & $\mathbf{X i}$ & (trial, YR score) \\
\hline QYr.niab-1A.1 & $1 \mathrm{~A}$ & 14.29 & 7.94 & -3.67 & -12.58 & 13.31 & 12.67 & 4.16 & 1 & NIAB15, S2 (cov0) \\
\hline QYr.niab-2A.1 & $2 \mathrm{~A}$ & 13.19 & 19.73 & 25.82 & 11.65 & 3.46 & 19.59 & 9.29 & 1 & OSG15, S2 (cov10) \\
\hline QYr.niab-2B.1 & $2 \mathrm{~B}$ & -5.48 & 0.72 & 6.79 & -6.41 & -12.6 & 1.97 & -33.82 & 1 & NIAB15, S2 $(\operatorname{cov} 10)$ \\
\hline QYr.niab-2D.1 & $2 \mathrm{D}$ & -21.77 & 13.46 & -16.70 & -5.12 & 33.03 & 21.44 & 12.13 & 1 & NIAB15, S2 $(\operatorname{cov} 10)$ \\
\hline QYr.niab-3A.1 & $3 \mathrm{~A}$ & 1.12 & -4.78 & -6.15 & -16.79 & -22 & -5.16 & -6.81 & 1 & ROTH15, S2 (cov0) \\
\hline QYr.niab-6A.1 & $6 \mathrm{~A}$ & 16.38 & 14.57 & 14.2 & 7.04 & 20.69 & 21.6 & 7.81 & 1 & NIAB15, S2 $(\operatorname{cov} 10)$ \\
\hline
\end{tabular}

949

Table 4. Predicted MAGIC founder effects at six of the eight 'major' yellow rust (YR) resistance QTL. Founder effects presented are from the 2015 trials, and correspond to the consensus marker with the most significant $p$-value identified with QTL mapping methods 2 , (identity by descent), 3 (interval mapping, cov=0) or 4 (composite interval mapping, covariates $=10$ ). For QTL mapping method 2 , contributions are relative to Alchemy whereas for methods 3 and 4, they are relative to Xi19. This was calibrated by adding an arbitrary value of 1 to all founder effects. For QYr.niab-2B.1 and QYr.niab-2D.1, founder effects represented are from the peak marker with the highest \% variation explained. QTL identified with IBS or IBD analyses only (QYr.niab-2A.2, QYr.niab-6A.2) are not included. 


\begin{tabular}{llllll}
\hline & SS & df & MS & $\boldsymbol{F}$ & $\boldsymbol{p}$ \\
\hline Interactions & 27177.9 & 15 & 1811.9 & 47.2 & $2.2 \mathrm{E}^{-16}$ \\
Residuals & 2377.7 & 62 & 38.4 & & \\
\hline
\end{tabular}

958

959

960

961

962

Table 5. Analysis of variance (ANOVA) in QTL interactions in response to yellow rust disease severity. SS $=$ sum of squares. Df $=$ degrees of freedom. $\mathrm{MS}=$ mean squares 


\section{Figures}
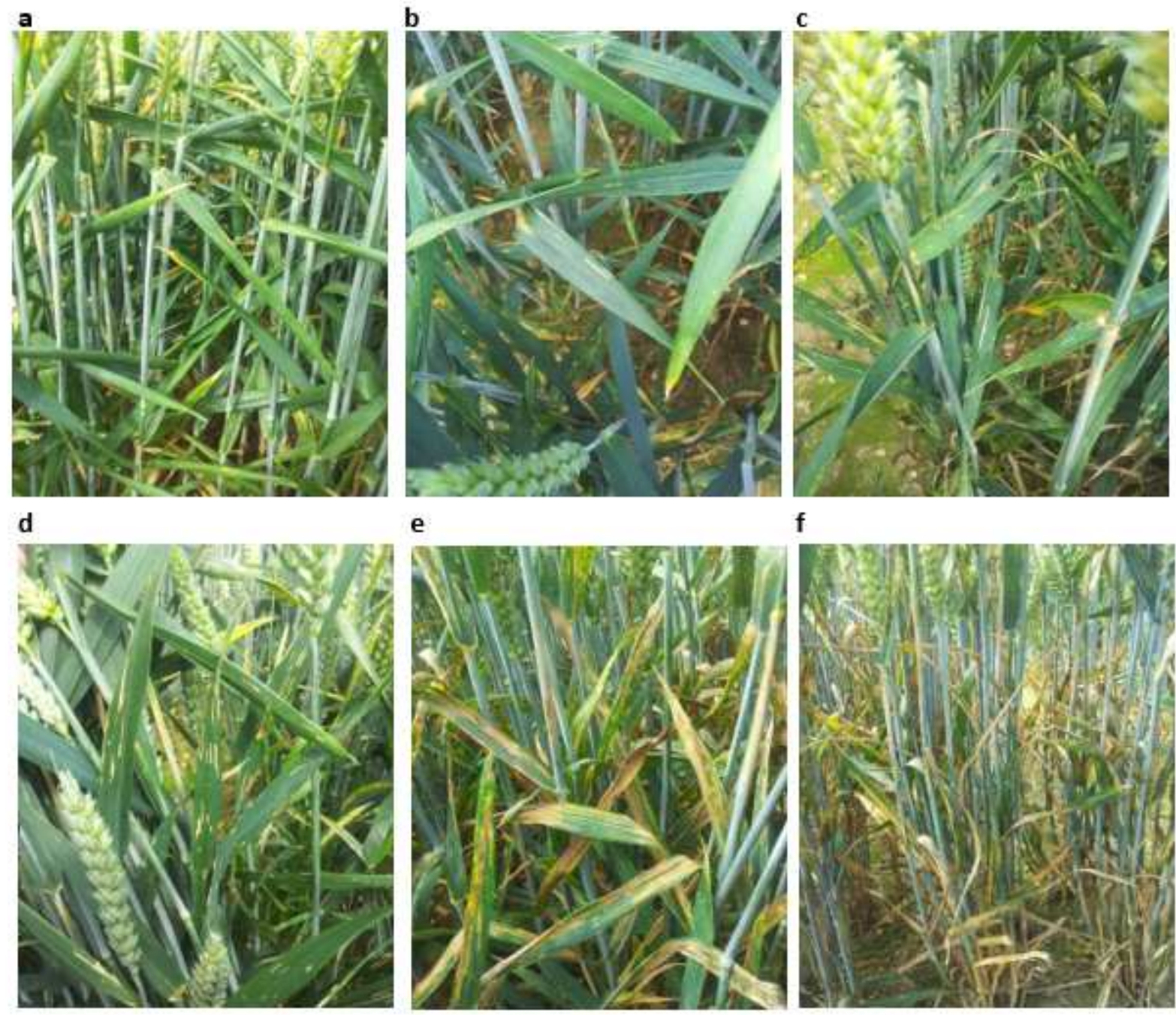

e

f
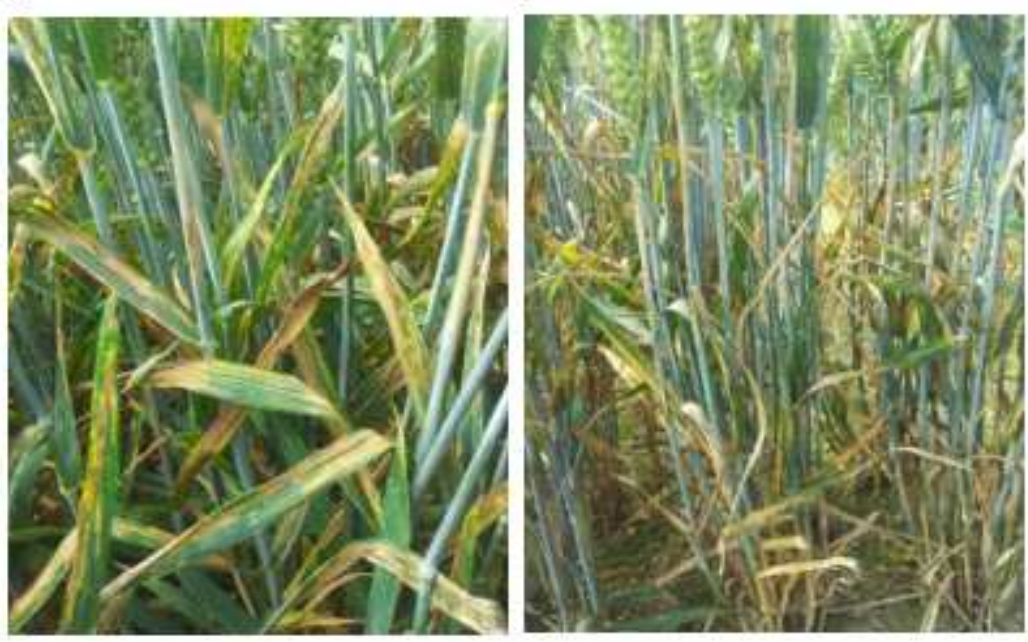

Figure 1

Examples of yellow rust resistance phenotypes in the MAGIC population. Assessments were made using the modified Cobb's scale (Peterson et al. 1948) ranging from 0 to $100 \%$ : (a) score $=0$, no infection, $0 \%$, (b) some tillers infected with a few stripes $0-15 \%$, (c) most tillers infected with several stripes, $15-25 \%$, (d) all leaves infected, $25-50 \%$, (e) all leaves infected, $50-75 \%$, (f) little to no green tissue left, $>75 \%$. 


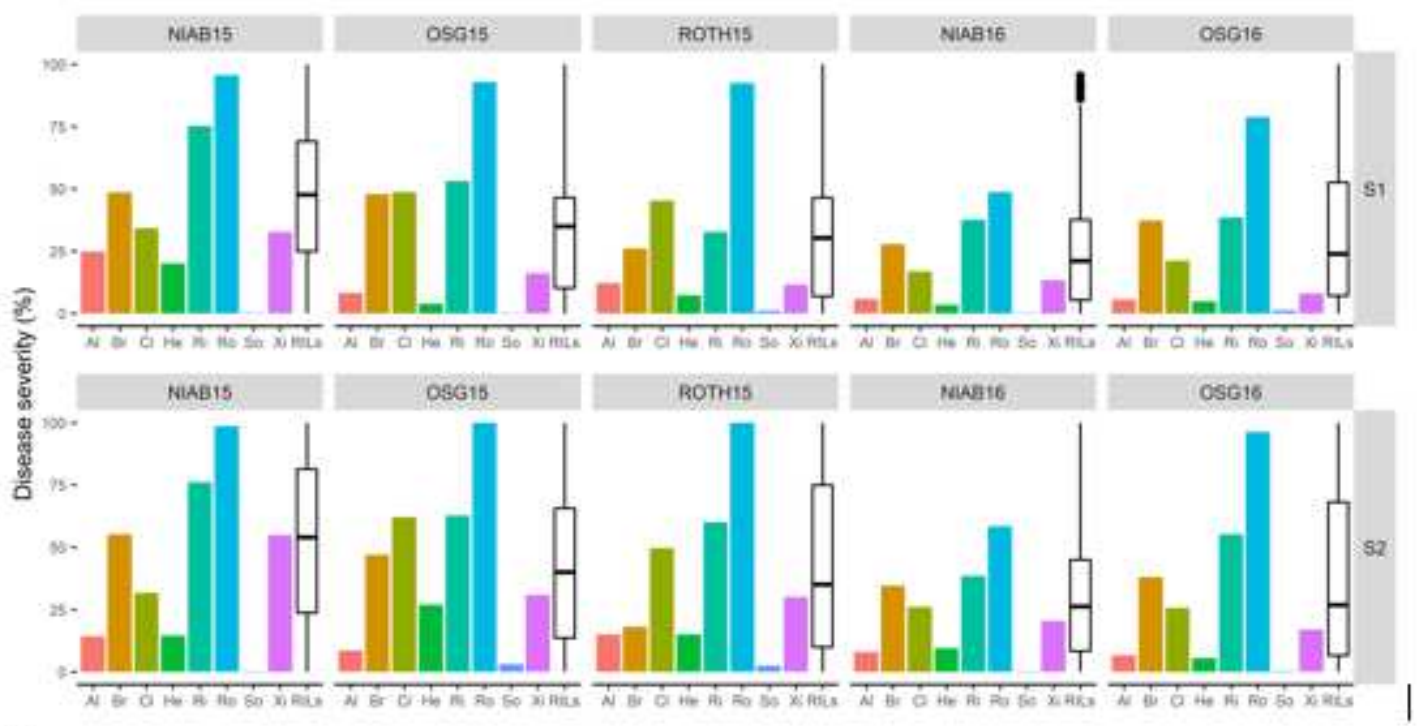

b
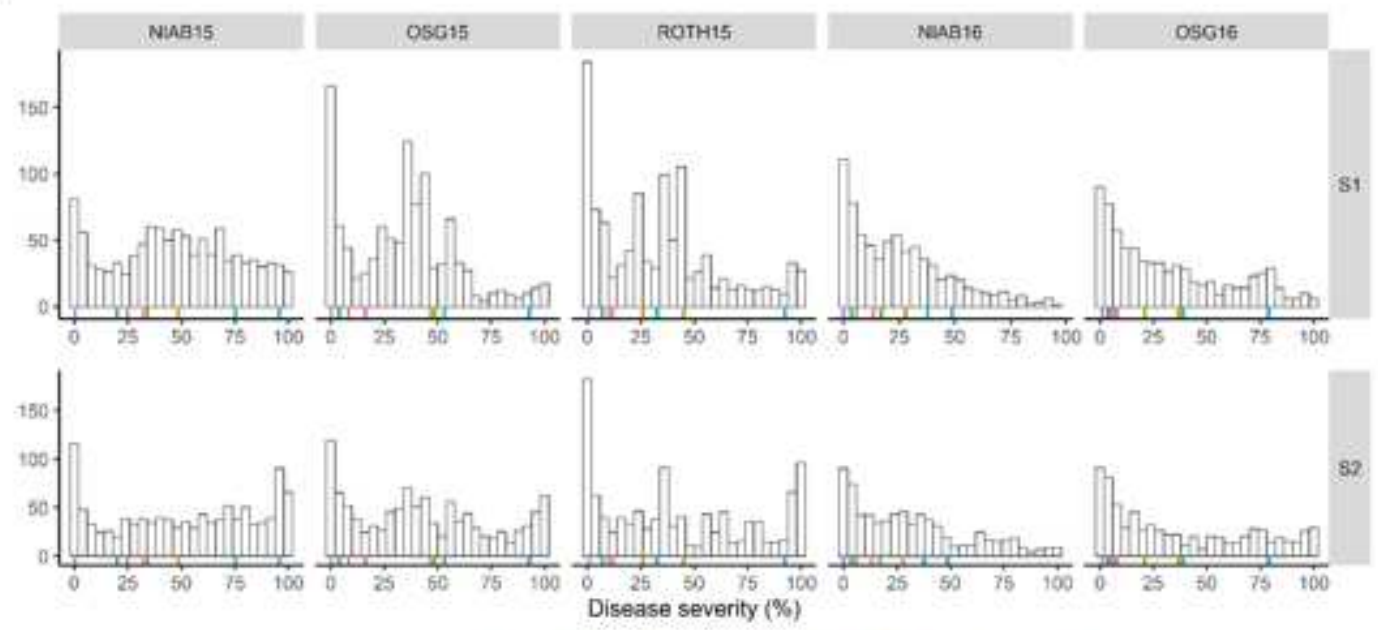

$\frac{n}{\underline{\underline{x}}}$

MAGIC Founders $\mid$ Al $\mid$ be $\mid$ Cl $\mid$ He $\mid$ Bi $\mid$ Ho $\mid$ so $\mid$ X

\section{Figure 2}

Yellow rust resistance in the MAIGC population. Five test environments: NIAB, Osgodby and Rothwell in 2015 (NIAB15, OSG15, ROTH15) and at NIAB and Osgodby in 2016 (NIAB16, OSG16). At each site, YR infection was recorded at two timepoints: S1 (score 1) and S2 (score 2). Al = Alchemy, $\mathrm{Br}=\mathrm{Brompton}, \mathrm{Cl}=$ Claire, $\mathrm{He}=$ Hereward, $\mathrm{Ri}=$ Rialto, $\mathrm{Ro}=$ Robigus, $\mathrm{So}=$ Soissons, $\mathrm{Xi}=\mathrm{Xi19}$. (A) MAGIC founder percentage YR resistance scores. Additionally, boxplots for all MAGIC recombinant inbred lines are indicated for each trial. (B) Histograms of percentage YR infection in the MAGIC recombinant inbred lines (RILs). Data for the non-transformed adjusted means (BLUEs) are shown for the RILs,. BLUEs for each of the eight founders are overlaid below, following the colour coding shown in the key. Note: for QTL analysis, the 2016 season data was log transformed. Negative values $=0$. Values over $100=100$. 

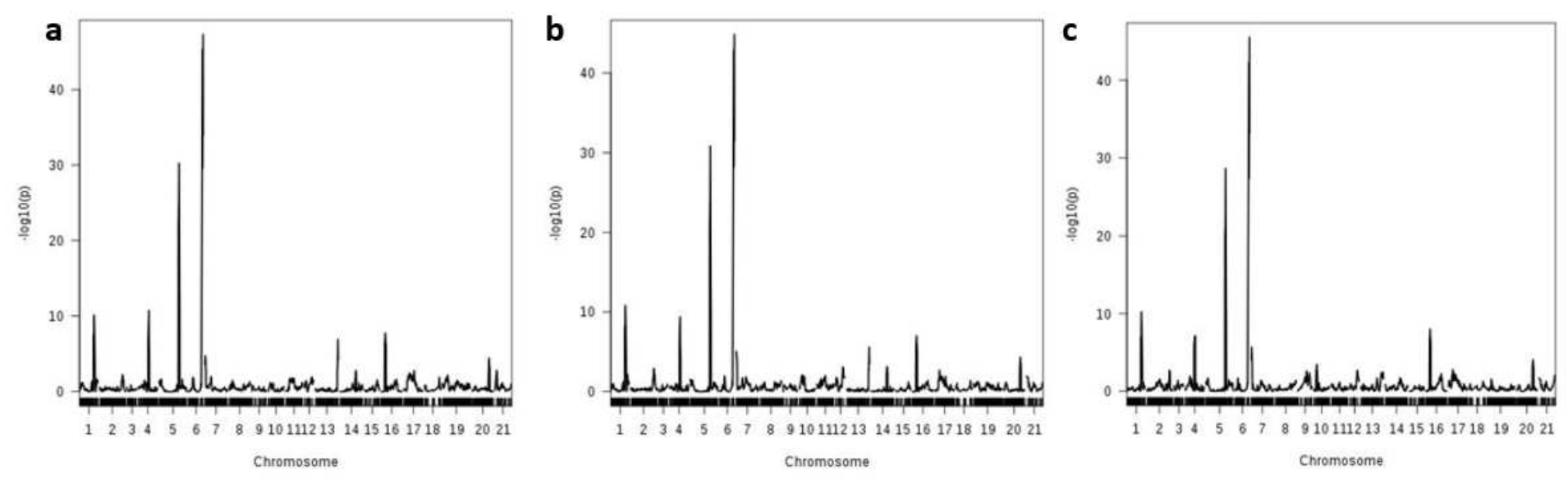

\section{Figure 3}

Examples of yellow rust resistance genetic analysis. Shown is the NIAB15 dataset analysed using composite interval mapping with 10 covariates (CIM-cov10), showing QTL scans using the following phenotypes: (A) scoring time-point 1, (B) scoring time-point 1, and (C) scoring time-point 2. All 21 wheat chromosomes are shown, with $1=$ chromosome $1 \mathrm{~A}, 2=1 \mathrm{~B}, 3=1 \mathrm{D}$, through to $21=7 \mathrm{D}$. 

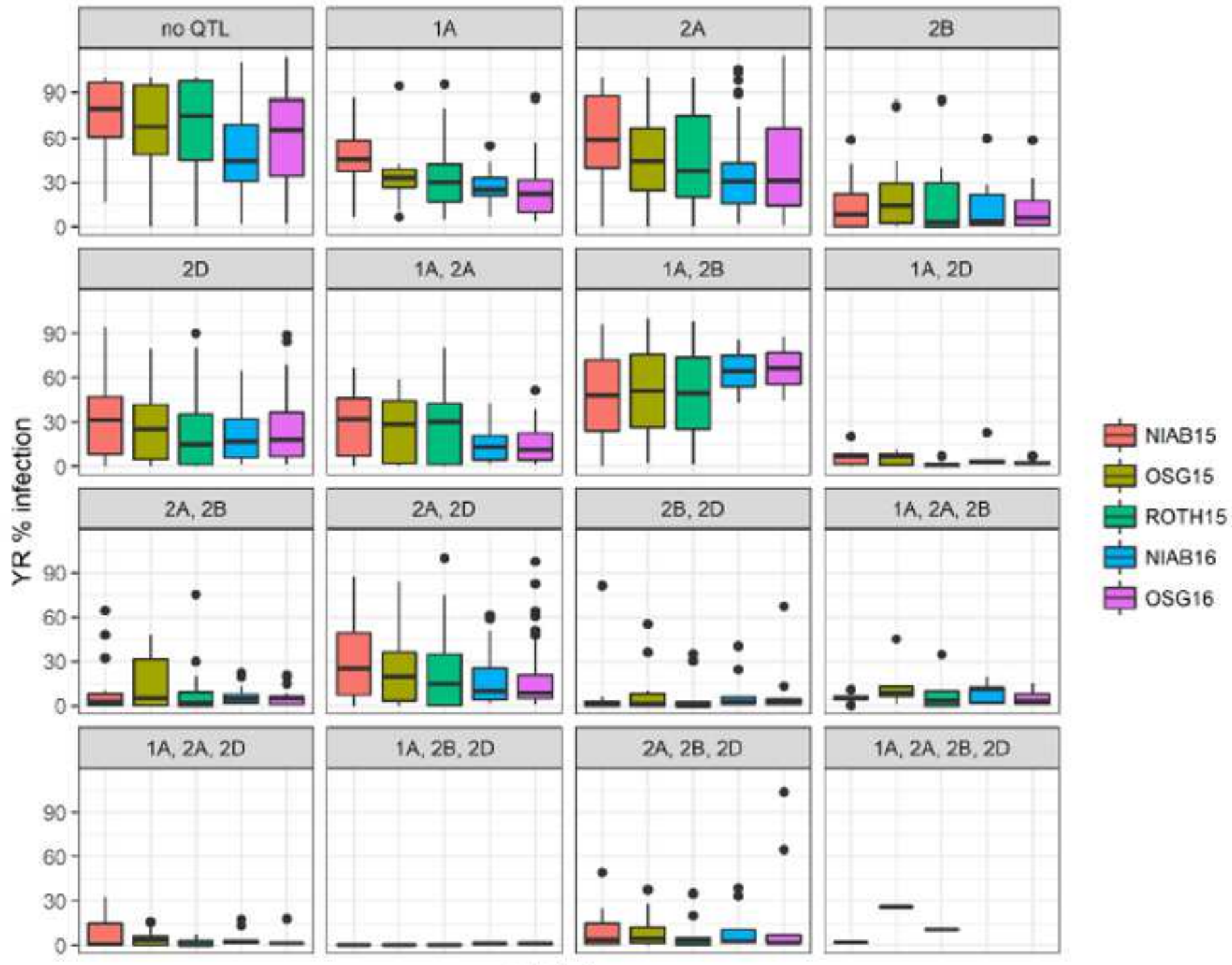

Trial sites

Figure 4

The effects of combining yellow rust (YR) resistance alleles at multiple QTL. Boxplots of the distribution of YR percentage infection in MAGIC recombinant inbred lines (RILs) in each of the five field trials. Based on the presence of resistance alleles at up to four of the most significant MAGIC YR resistance quantitative trait loci (QTL): on chromosomes 1A (QYr.niab-1A.1), 2A (QYr.niab-2A.1), 2B (QYr.niab-2B.1) and 2D (QYr.niab-2D.1). Boxes denote the interquartile range, the horizontal lines that bisect them the median, the vertical lines that extend from them the $1.5 \mathrm{x}$ interquartile range beyond it, and the dots the outlier MAGIC RILs. 

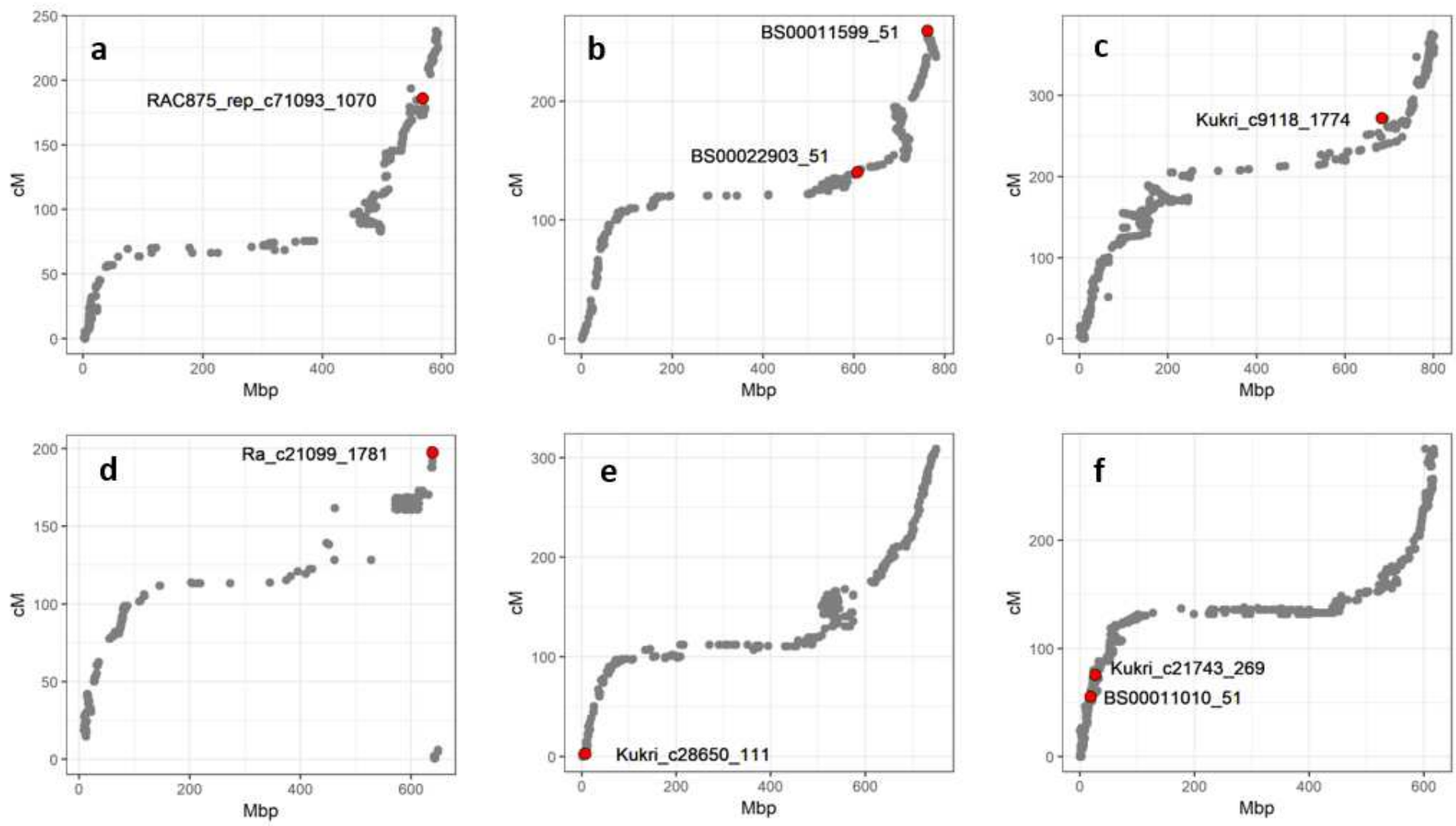

\section{Figure 5}

Locations of the eight most significant yellow rust QTL visualised on plots of all genetically mapped SNPs in the MAGIC genetic map (cM) (Gardner et al. 2016) versus their physical map locations (Mbp) (IWGSC Refseq v1.0). Shown are: (A) QYr.niab-1A.1 (represented by peak SNP RAC875_rep_c71093_1070) on chromosome 1A, (B) QYr.niab-2A.1 (BS00022903_51) and QYr.niab-2A.2 (BS00011599_51) on chromosome 2A. (C) QYr.niab-2B.1 (Kukri_c9118_1774) on chromosome 2B. (D) QYr.niab-2D.1 (Ra_c21099_1781) on chromosome 2D. (E) QYr.niab-3A.1 (Kukri_c28650_111) on chromosome 3A. (F) QYr.niab-6A.1 (BS00011010_51) and QYr.niab-6A.2 (Kukri_c21743_269) on chromosome 6A. 


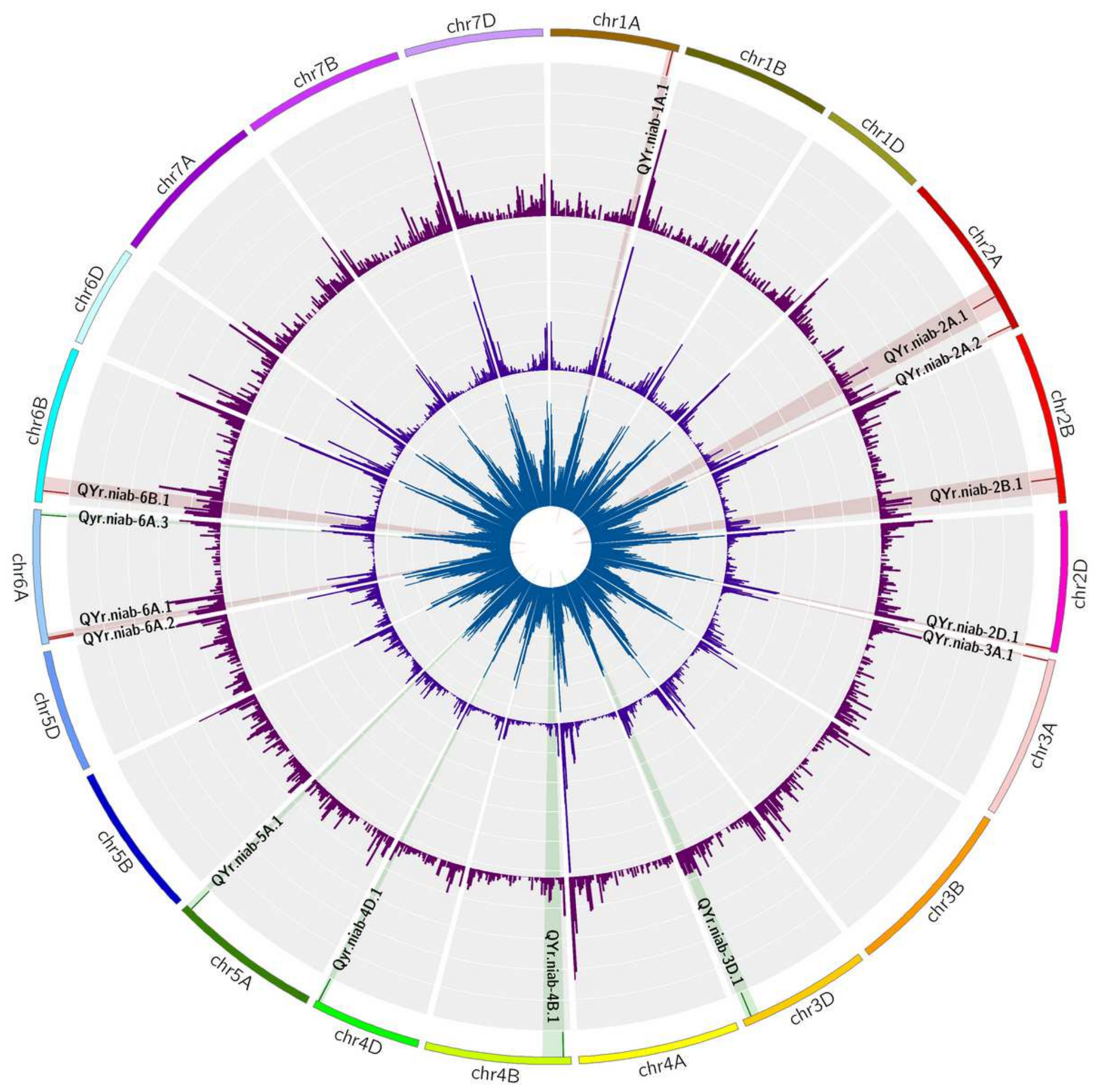

Figure 6

Circular diagram representing the position of 14 yellow rust (YR) resistance QTL identified in the MAGIC wheat population, in relation to density of candidate 'resistance genes' present in the cv. Chinese Spring reference genome (RefSeq v1.0; IWGSC, 2018). Starting from the outermost, Track 1: chromosome number. Track 2: tick marks indicating the position of major (red) and minor (green) YR QTL. Track 3 (purple): histogram of the proportion of resistant genes per gene per 10Mbp. Track 4 (blue): histogram of 'resistant gene' count every $10 \mathrm{Mbp}$. Track 5 (dark blue): histogram of gene count every $10 \mathrm{Mbp}$. 
a

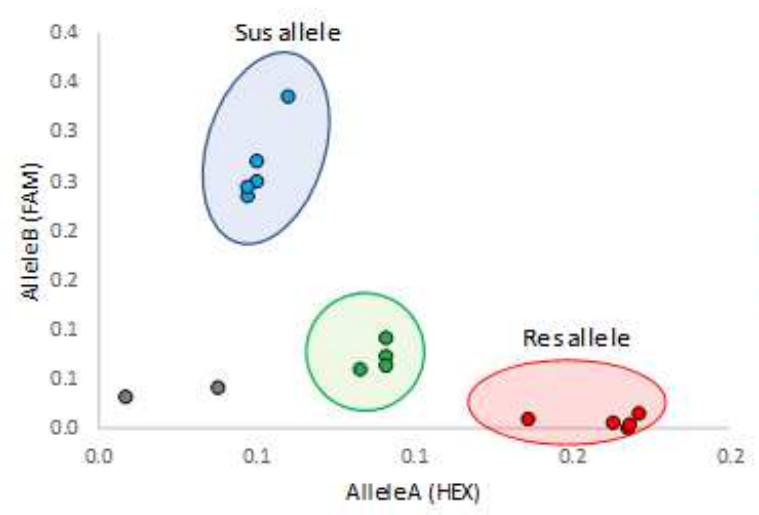

C

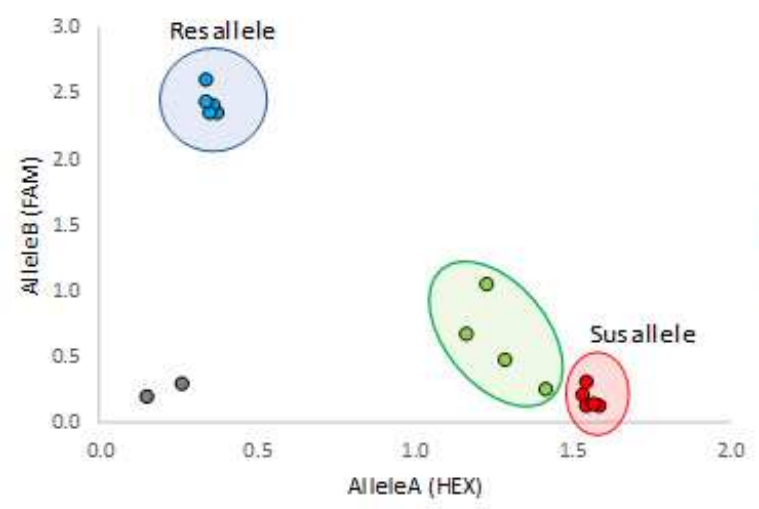

b

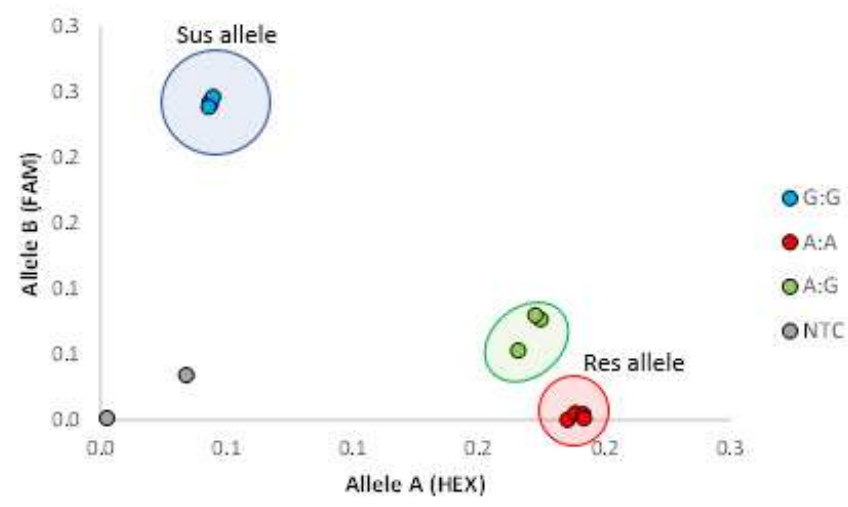

d

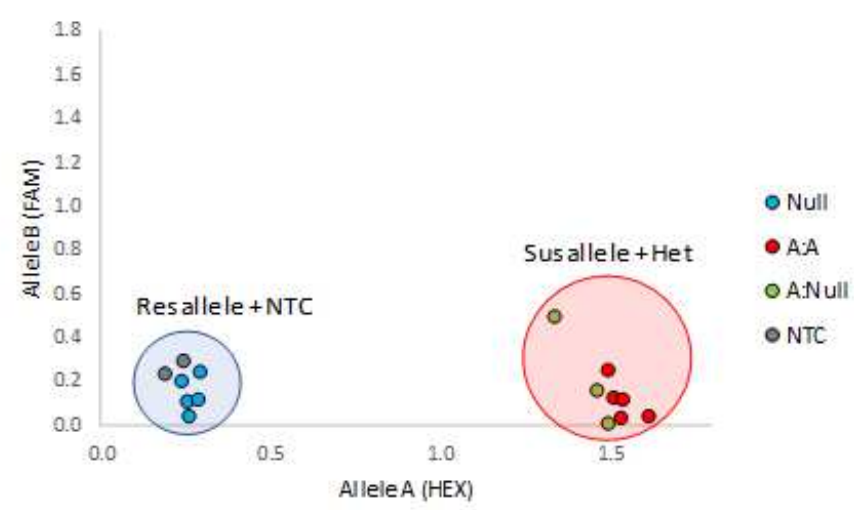

\section{Figure 7}

Examples of genotyping results for some of the KASP markers developed for the four most significant yellow rust resistance QTL (see Supplementary Table 2 for full details). Genotype calls for resistant (Res) and susceptible (Sus) alleles are indicated, derived from genomic DNA template from the founders subsequently indicated here. NTC = no template negative control. (A) QYr.niab-1A.1: KASP marker tplb0021i12_383 (DNA: Alchemy, susceptible allele; Hereward, resistant allele). (B) QYr.niab-2A.1: KASP marker BS00062679_51 (DNA: Xi19, resistant allele; Claire, susceptible allele). (C) QYr.niab-2B.1: KASP marker BS00016650_51 (DNA: Soissons, resistant allele; Robigus, susceptible allele). (D) QYr.niab-2D.1: KASP marker Kukri_c498_2381 (DNA: Claire, resistant allele; Soissons, susceptible allele).

\section{Supplementary Files}

This is a list of supplementary files associated with this preprint. Click to download.

- SupplementaryTable1.docx

- SupplementaryTable2.xIsx

- SupplementaryTable3.docx

- SupplementaryTable4.xlsx 
- SupplementaryTable5.docx

- SupplementaryTable6.docx

- SupplementaryTable7.docx

- SupplementaryTable8.xlsx

- SupplementaryTable9.docx

- SupplementaryText.docx 